\title{
CHEBYSHEV POLYNOMIALS \\ IN SEVERAL VARIABLES AND THE RADIAL PART OF THE LAPLACE-BELTRAMI OPERATOR
}

\author{
R. J. BEERENDS
}

\begin{abstract}
Chebyshev polynomials of the first and the second kind in $n$ variables $z_{1}, z_{2}, \ldots, z_{n}$ are introduced. The variables $z_{1}, z_{2}, \ldots, z_{n}$ are the characters of the representations of $S L(n+1, \mathrm{C})$ corresponding to the fundamental weights. The Chebyshev polynomials are eigenpolynomials of a second order linear partial differential operator which is in fact the radial part of the Laplace-Beltrami operator on certain symmetric spaces. We give an explicit expression of this operator in the coordinates $z_{1}, z_{2}, \ldots, z_{n}$ and then show how many results in the literature on differential equations satisfied by Chebyshev polynomials in several variables follow immediately from well-known results on the radial part of the Laplace-Beltrami operator. Related topics like orthogonality, symmetry relations, generating functions and recurrence relations are also discussed. Finally we note that the Chebyshev polynomials are a special case of a more general class of orthogonal polynomials in several variables.
\end{abstract}

\section{INTRODUCTION}

In recent years quite a number of papers on Chebyshev polynomials in several variables have appeared. We divide these papers in two groups, namely the papers by Bacry [1, 2, 3] and the papers by Lidl [19], Dunn and Lidl [11, 12], Eier and Lidl [14], Eier, Lidl and Dunn [13] and also Ricci [23] (including some of the references given in these papers). Both groups of papers consider Chebyshev polynomials of the first and the second kind. In the two variable case they occur as special cases $\left(\alpha= \pm \frac{1}{2}\right)$ in Koornwinder [18, III-IV], which is the first systematic treatment of classes of orthogonal polynomials associated with root systems. They are also special cases $(\gamma=0,1)$ in Debiard [7], where Koornwinder's results are generalized to several variables (but no proofs are given). An important property of the polynomials is the fact that they are eigenfunctions of certain partial differential operators (cf. [18, 7]). One of these operators is of second order and for certain values of the parameter involved (e.g. for the Chebyshev cases) it is the radial part of the Laplace-

Received by the editors September 15, 1988 and, in revised form, October 20, 1989.

1980 Mathematics Subject Classification (1985 Revision). Primary 22E30; Secondary 33A65, 33A75, 43A75.

Research supported partially by a grant from the Netherlands organization for the advancement of pure research (Z.W.O.). 
Beltrami operator on certain symmetric spaces. The main point of the present paper is to show that the $n$-dimensional results in $[1,2,3,19,13]$ on differential equations satisfied by Chebyshev polynomials all follow from results on the radial part of the Laplace-Beltrami operator on certain symmetric spaces of rank $n$. Here [4, Proposition 8] plays a crucial role. We first concentrate on Chebyshev polynomials of the second kind.

In the papers by Bacry, Chebyshev polynomials of the second kind are defined as characters of $S L(n+1, \mathrm{C})$, which can be parametrized by partitions $\lambda=\left(\lambda_{1}, \lambda_{2}, \ldots, \lambda_{n}\right) \quad\left(\lambda_{i}\right.$ integers and $\left.\lambda_{1} \geq \lambda_{2} \geq \cdots \geq \lambda_{n} \geq 0\right)$. He states that they are eigenfunctions of a certain second order partial differential operator. This differential operator has homogeneous polynomials $B_{k, l}$ of degree two in the elementary symmetric polynomials as coefficients. The $B_{k, l}$ are defined using the so-called Laguerre-emanent. In $\S 3$ we define a second order partial differential operator $L(m)$ involving a complex parameter $m$. For certain integer values of $m, L(m)$ is the radial part of the Laplace-Beltrami operator on certain symmetric spaces. If $m=2$ (this corresponds to $\alpha=\frac{1}{2}$ and $\gamma=1$ above) then $L(2)$ corresponds to $S L(n+1, \mathrm{C}) / S U(n+1)$ in the noncompact case and to $S U(n+1)$, considered as symmetric space, in the compact case. $L(0)$ is the ordinary Laplacian on a Euclidean space (a flat symmetric space). In [4, Proposition 8] we obtained $L(m)$ in certain coordinates, which we will call the " $z$-coordinates", and for convenience we state this result in $\S 3$. In fact these $z$-coordinates are the characters of the representations of $S L(n+1, \mathrm{C})$ corresponding to the fundamental weights (cf. $\S 3)$. In $\S 4$ we then show that Bacry's differential operator is precisely $L(2)$ in the $z$-coordinates. Since characters are eigenfunctions of $L(2)$ it follows immediately that Chebyshev polynomials of the second kind are eigenfunctions of $L(2)$, thus proving [2, IV.b], [3, (27)-(29)] and answering the open problem [2, V.1] (see §5.b). Bacry pointed out to me that the $B_{k, l}$ should be the same as the coefficients of the so-called Bezoutian in Weber's book [28, $\S 79,(8)]$. It was not obvious to me that Weber's definition agreed with the one given by Bacry and we have also not been able to find an explicit expression for the coefficients of the Bezoutian in the literature (for small values of $n$ they occur in $[28, \S 79]$ ). Therefore we give such an explicit expression in $\S 4$ by showing that they are indeed the coefficients in the second order part of $L(m)$ in the $z$-coordinates. From the orthogonality of the characters we also obtain Bacry's orthogonality result [2, IV.c], [3, (25)] (see $\S 5 . c)$.

In $\S 5$.a we give a precise definition of Chebyshev polynomials of the second kind $U_{\lambda}, \lambda=\left(\lambda_{1}, \lambda_{2}, \ldots, \lambda_{n}\right) \quad\left(\lambda_{i}\right.$ integers $)$, and we show the relation with other definitions and special cases in the second group of papers mentioned above. In particular we show that the polynomials $P_{k, l}^{1 / 2}$ in $[11,12,13]$ are the special case $\lambda=(k+l, l, \ldots, l)$. The case $l=0$ was studied in $[19,23]$. However, in all these papers the connection with characters of $S L(n+1, \mathbf{C})$ is not made. The second order partial differential equation for the $P_{k, l}^{1 / 2}$ given 
in [13, Theorem 2] (and for the case $l=0$ in [19, (40)]) involves once more the operator $L(2)$ in the $z$-coordinates so that their results again follow from the differential equation satisfied by characters of $S L(n+1, \mathrm{C})$ (see $\S 5 . b$ ). Orthogonality is not considered in this second group of papers (but see [14, $\S 5])$.

In $\S 6$ we turn our attention to Chebyshev polynomials of the first kind which are essentially symmetric sums of exponential monomials (see $\S 6 . a$ ). It follows immediately that these are eigenfunctions of the operator $L(0)$, which is simply the ordinary Laplacian on a Euclidean space. From the expression of $L(0)$ in the $z$-coordinates all the results on differential equations satisfied by Chebyshev polynomials of the first kind in $[1,2,3,19,13]$ follow (see $\S 6 . b$ ). In $\S 6 . c$ we treat the orthogonality results in [14], [2, V.2.c] and answer the open problem [2, V.2].

Although our main point concerns differential equations and orthogonality for Chebyshev polynomials, we also observed that many results on symmetry relations, generating functions and recurrence relations in the above mentioned papers could be obtained from known results on characters of $S L(n+1, \mathbf{C})$ and related topics. It seemed appropriate to include some remarks on these matters in the present paper (mainly in $\S \S 5 . d$ and 6.d).

The Chebyshev polynomials treated in this paper are associated with a root system of type $A_{n}$ for the values $m=0$ and 2 (cf. $\S 2 . c$ ). The last section contains some remarks on generalizations to other values of the parameter $m$ (which do not necessarily correspond to a symmetric space) and to other types of root systems; a subject where quite a few new results have been obtained very recently.

\section{NOTATIONS AND PRELIMINARIES}

a. Symmetric polynomials. The reference for this subsection is Macdonald [20]. We use largely the same notation. Consider the ring $\mathbf{Z}\left[x_{1}, \ldots, x_{n}\right]$ of polynomials in $n$ independent variables with integer coefficients. The symmetric group $S_{n}$ acts on $\mathbf{Z}\left[x_{1}, \ldots, x_{n}\right]$ by permuting the variables. Let $\Lambda_{n}$ be the subring of symmetric polynomials [20, I.2]. If $\alpha=\left(\alpha_{1}, \ldots, \alpha_{n}\right) \in\left(\mathbf{Z}^{+}\right)^{n}$ then we put $|\alpha|=\alpha_{1}+\cdots+\alpha_{n}$ and we let $x^{\alpha}$ denote the monomial $x_{1}^{\alpha_{1}} \cdots x_{n}^{\alpha_{n}}$. A partition $\lambda$ is a sequence $\lambda=\left(\lambda_{1}, \lambda_{2}, \ldots, \lambda_{r}\right)$ such that $\lambda_{1} \geq \lambda_{2} \geq \cdots \geq \lambda_{r} \geq 0$. Note that we allow the $\lambda_{i}$ to be zero. The number of parts, i.e. the number of nonzero $\lambda_{i}$, is called the length of $\lambda$ and is denoted by $l(\lambda)$. We also use the notation $\lambda=\left(1^{\mu_{1}} 2^{\mu_{2}} \cdots s^{\mu_{s}}\right)$ if exactly $\mu_{j}$ of the parts of $\lambda$ are equal to $j$. The set of all partitions is denoted by $\mathscr{P}$. Given a partition $\lambda$ we define the dual partition $\lambda^{\prime}=\left(\lambda_{1}^{\prime}, \ldots, \lambda_{s}^{\prime}\right)$ by $\lambda_{i}^{\prime}=\operatorname{card}\left\{j: \lambda_{j} \geq i\right\}$. For a partition $\lambda$ of length $\leq n$ we define a symmetric polynomial $m_{\lambda}$ by

$$
m_{\lambda}=m_{\lambda}\left(x_{1}, \ldots, x_{n}\right)=\sum x^{\alpha},
$$

where the sum is taken over all distinct permutations $\alpha$ of $\lambda$. For each integer 
$r \geq 0$ the $r$ th elementary symmetric polynomial $e_{r}$ is defined by

$$
e_{0}=1, \quad e_{r}=\sum_{1 \leq i_{1}<\cdots<i_{r} \leq n} x_{i_{1}} x_{i_{2}} \cdots x_{i_{n}}=m_{\left(1^{r}\right)} .
$$

We also define $e_{r}=0$ if $r>n$ and $r<0$. The $e_{r}$ are the coefficients of the polynomial

$$
E(t)=\prod_{i=1}^{n}\left(1+x_{i} t\right)=\sum_{r=0}^{n} e_{r} t^{r} .
$$

Next we define for each integer $r \geq 0$ the $r$ th complete symmetric function $h_{r}$ by

$$
h_{r}=\sum_{|\lambda|=r} m_{\lambda}
$$

The generating function for the $h_{r}$ is

$$
H(t)=\sum_{r \geq 0} h_{r} t^{r}=\prod_{i=1}^{n}\left(1-x_{i} t\right)^{-1} .
$$

Note that $H(t) E(-t)=1$. The power sums $p_{r}$ are defined for each integer $r \geq 1$ by

$$
p_{r}=\sum_{i=1}^{n} x_{i}^{r}=m_{(r)},
$$

and the generating function for the $p_{r}$ is $\left[20,(2.10)-(2.10)^{\prime}\right]$

$$
P(t)=\sum_{r \geq 1} p_{r} t^{r-1}=H^{\prime}(t) / H(t)=E^{\prime}(-t) / E(-t),
$$

from which we obtain

$$
k h_{k}=\sum_{r=1}^{k} p_{r} h_{k-r}, \quad k \geq 1,
$$

or equivalently (use the involution $\omega$ as defined in $[20, \S 2]$ )

$$
k e_{k}=\sum_{r=1}^{k}(-1)^{r-1} p_{r} e_{k-r}, \quad k \geq 1,
$$

which are the so-called Newton identities.

A polynomial $p$ in $\mathbf{Z}\left[x_{1}, \ldots, x_{n}\right]$ is called skew-symmetric if $w p=\varepsilon(w) p$ for all $w \in S_{n}$, where $\varepsilon(w)$ denotes the sign of the permutation $w$. Let $a_{\alpha}$ be the skew-symmetric polynomial given by

$$
a_{\alpha}=a_{\alpha}\left(x_{1}, \ldots, x_{n}\right)=\sum_{w \in S_{n}} \varepsilon(w) \cdot w\left(x^{\alpha}\right) .
$$

Since $a_{\alpha}$ vanishes unless $\alpha_{1}, \ldots, \alpha_{n}$ are all distinct we may assume that $\alpha_{1}>$ $\alpha_{2}>\cdots>\alpha_{n} \geq 0$ and thus $\alpha=\lambda+\rho$ where

$$
\rho=(n-1, n-2, \ldots, 1,0) \text {. }
$$


Then

$$
a_{\alpha}=a_{\lambda+\rho}=\sum_{w \in S_{n}} \varepsilon(w) \cdot w\left(x^{\lambda+\rho}\right)=\operatorname{det}\left(x_{i}^{\lambda_{j}+n-j}\right)_{1 \leq i, j \leq n} .
$$

Note that $a_{\rho}$ is the well-known Vandermonde determinant

$$
a_{\rho}=\operatorname{det}\left(x_{i}^{n-j}\right)=\prod_{1 \leq i<j \leq n}\left(x_{i}-x_{j}\right) .
$$

Each $a_{\lambda+\rho}$ is divisible by $a_{\rho}$ in $\mathbf{Z}\left[x_{1}, \ldots, x_{n}\right][20, \S 3]$ and the quotient is a symmetric polynomial. Put

$$
s_{\lambda}=s_{\lambda}\left(x_{1}, \ldots, x_{n}\right)=a_{\lambda+\rho} / a_{\rho} .
$$

The following formulas, known as Jacobi-Trudi formulas, express the $s_{\lambda}$ as polynomials in the $e_{r}$ and $h_{r}[20,(3.5)]$

$$
s_{\lambda}=\operatorname{det}\left(e_{\lambda_{i}^{\prime}-i+j}\right)_{1 \leq i, j \leq l\left(\lambda^{\prime}\right)}=\operatorname{det}\left(h_{\lambda_{i}-i+j}\right)_{1 \leq i, j \leq l(\lambda)} \text {. }
$$

In particular

$$
s_{\left(1^{r}\right)}=e_{r}, \quad s_{(r)}=h_{r}=\operatorname{det}\left(e_{1-i+j}\right)_{1 \leq i, j \leq r} .
$$

Next we recall some results on expansions related to $H(t)$. Let $x=\left(x_{1}, \ldots, x_{n}\right)$ and $y=\left(y_{1}, \ldots, y_{n}\right)$ be two sets of independent variables. Denote symmetric functions of $x$ (resp. $y$ ) by $s_{\lambda}(x), p_{\lambda}(x)$, etc. (resp. $s_{\lambda}(y), p_{\lambda}(y)$, etc.). Then one has $[20,(4.2)]$

$$
\prod_{i, j=1}^{n}\left(1-x_{i} y_{j}\right)^{-1}=\sum_{\lambda} h_{\lambda}(x) m_{\lambda}(y)
$$

where $h_{\lambda}=h_{\lambda_{1}} h_{\lambda_{2}} \cdots h_{\lambda_{r}}$ if $\lambda=\left(\lambda_{1}, \ldots, \lambda_{r}\right)$ and the sum is taken over all partitions of length $\leq n$. From the proof of [20, (4.3)] (which uses (2.10) and (2.12)) we extract the following result

$$
a_{\rho}(x) \prod_{i, j=1}^{n}\left(1-y_{i} x_{j}\right)^{-1}=\sum_{\alpha \in\left(\mathbf{Z}^{+}\right)^{n}} s_{\alpha-\rho}(y) x^{\alpha} .
$$

b. Complex semisimple Lie groups. The reference for this subsection is Helgason [16, 17], mainly Chapter VII in [16] and Chapter V in [17]. Let $G$ be a connected, simply connected complex semisimple Lie group with Lie algebra $\mathfrak{g}$. Consider a compact real form $\mathfrak{u}$ of $\mathfrak{g}$ and let $U$ be the analytic subgroup of $G$ corresponding to $\mathfrak{u}$. Then $U$ is simply connected (follows from Weyl's theorem [16, Chapter II, Theorem 6.9]; see also [26, Theorem 4.11.14]). Let $T \subset U$ be a maximal torus in $U$ and $\mathfrak{t} \subset \mathfrak{u}$ the Lie algebra of $T$. The subalgebra of $\mathfrak{g}$ generated by $\mathfrak{t}$ will be denoteld by $\mathfrak{h}$. Then $\mathfrak{h}$ is a Cartan subalgebra of $\mathfrak{g}$ and so we can consider the set $\Delta$ of nonzero roots of $\mathfrak{g}$ with respect to $\mathfrak{h}$. Let $\langle\cdot, \cdot\rangle$ denote the bilinear form on $\mathfrak{h}$ induced by the Killing form of $\mathfrak{g}$. If $\mu \in \mathfrak{h}^{*}$, the dual of $\mathfrak{h}$, then $H_{\mu} \in \mathfrak{h}$ is determined by $\left\langle H, H_{\mu}\right\rangle=\mu(H), H \in \mathfrak{h}$. Put $\langle\mu, \nu\rangle=\left\langle H_{\mu}, H_{\nu}\right\rangle, \mu, \nu \in \mathfrak{h}^{*}$. Let $\mathfrak{h}_{\mathbf{R}}=\sum_{\alpha \in \Delta} \mathbf{R} \cdot H_{\alpha}$, then $\mathfrak{t}=i \mathfrak{h}_{\mathbf{R}}$. 
Since roots are real-valued on $\mathfrak{h}_{\mathbf{R}}$ we may regard them as elements of the (real) dual $\mathfrak{h}_{\mathbf{R}}^{*}$ of the real vector space $\mathfrak{h}_{\mathbf{R}}$. Choose a Weyl chamber $\mathfrak{h}^{+}$in $\mathfrak{h}_{\mathbf{R}}$ and put $\mathfrak{t}^{+}=i \mathfrak{h}^{+}$. Denote by $\Delta^{+}$the corresponding set of positive roots. Let $\alpha_{1}, \ldots, \alpha_{n}$ denote the simple roots and $\omega_{1}, \ldots, \omega_{n}$ the dual basis given by $2\left\langle\omega_{j}, \alpha_{i}\right\rangle /\left\langle\alpha_{i}, \alpha_{i}\right\rangle=\delta_{i j} \quad(1 \leq i, j \leq n)$. Here $\delta_{i j}$ is the Kronecker delta. If $\Lambda$ denotes the weight lattice then $\Lambda=\sum_{i=1}^{n} \mathbf{Z} \omega_{j}$. If $\lambda \in \Lambda$ and $2\langle\lambda, \alpha\rangle /\langle\alpha, \alpha\rangle$ is nonnegative for all $\alpha \in \Delta^{+}$, then $\lambda$ is called a dominant weight. On $\Lambda$ we take the partial ordering defined by $\mathfrak{h}^{+}$, so that $\lambda \geq \mu$ if and only if $\lambda-\mu$ is a nonnegative linear combination of the simple roots. We also use the (compatible) notation $\alpha>0$ for a positive root $\alpha$. The Weyl group of $\Delta$ will be denoted by $W$. If $w \in W$ then $\varepsilon(w)$ denotes the determinant of $w$. Put $\rho=\frac{1}{2} \sum_{\alpha>0} \alpha$. Let $D(u)$ denote the diagram $\{H \in \mathfrak{t} \mid \alpha(H) \in 2 \pi i \mathbf{Z}$ for some $\alpha \in \Delta\}$ and put $\mathfrak{t}_{r}=\mathfrak{t}-D(u)$. Fix a component $P_{0}$ of $\mathfrak{t}_{r}$ whose closure contains the origin. We may assume that $P_{0}$ is inside $\mathrm{t}^{+}$.

As usual we identify $U$ with the Riemannian symmetric space of the compact type $U \times U / K$, where $K$ is the diagonal $\{(u, u) \mid u \in U\}$. Let $\chi_{\lambda}$ be the character of the irreducible representation of $U$ with highest weight $\lambda$. Since $\chi_{\lambda}$ is invariant under conjugation, it follows from [17, Chapter $\left.\mathrm{V}, \S 1,(16)\right]$ that $\chi_{\lambda}$ satisfies the linear partial differential equation (on $\exp P_{0}$ )

$$
\Delta\left(L_{U}\right) \chi_{\lambda}=(\langle\rho, \rho\rangle-\langle\lambda+\rho, \lambda+\rho\rangle) \chi_{\lambda} .
$$

Here $\Delta\left(L_{U}\right)$ denotes the radial part of the Laplacian $L_{U}$ on $U$ [17, Chapter II, Proposition 3.12]. For future purposes it is important to note that due to the identification of $U$ with $U \times U / K$ as above, we introduce a factor 2. The set of restricted roots of $(U \times U, K)$ corresponds to the roots of $\Delta$, but counted with multiplicity 2 . Consequently the linear function $\rho$ for the symmetric space $(U \times U, K)$ is twice the linear function $\rho$ arising from $\Delta$. The weight function in the integral formula for the Cartan decomposition, which enters in the explicit expression for $\Delta\left(L_{U}\right)$, has to be adjusted accordingly [17, Chapter I, $\S 5$, no. 2B and Chapter II, $\S 3$, no. 4, (v)].

By the Schur orthogonality relations we have

$$
\int_{U} \chi_{\lambda}(u) \overline{\chi_{\mu}(u)} d u=\delta_{\lambda, \mu},
$$

where the bar denotes complex conjugation and $\delta_{\lambda, \mu}=0$ if $\lambda \neq \mu$ and $=1$ if $\lambda=\mu$. Then a variation of Weyl's integration formula [17, Chapter I, Theorem 5.13] implies that

$$
\int_{P_{0}} \chi_{\lambda}(\exp H) \overline{\chi_{\mu}(\exp H)} \delta(\exp H) d H=c \cdot \delta_{\lambda, \mu},
$$

where $c$ is a constant and the weight function $\delta(\exp H)$ is given by

$$
\delta(\exp H)=\prod_{\alpha \in \Delta}\left(e^{\alpha(H) / 2}-e^{-\alpha(H) / 2}\right) .
$$


In the above integrations, the Haar measure $d u$ on $U$ is normalized by $\int d u=$ 1 and $d H$ is the Euclidean measure on $t$ induced by the Killing form. Since

$$
\delta(\exp H)=\prod_{\alpha>0}\left|e^{\alpha(H) / 2}-e^{-\alpha(H) / 2}\right|^{2}
$$

we have

$$
\delta(\exp H)^{1 / 2}=\prod_{\alpha>0}\left|e^{\alpha(H) / 2}-e^{-\alpha(H) / 2}\right| .
$$

Note that if $H \in P_{0}$ then $\delta(\exp H)>0$. We also recall the Peter-Weyl theorem which states that characters of irreducible representations form a complete orthogonal system in the space of all continuous class functions (a class function is a function invariant under conjugation $\left.x \rightarrow u x u^{-1}\right)$.

c. $S L(n+1, \mathbf{C})$

We now specialize to the case $G=S L(n+1, \mathbf{C})$, the group of all $(n+$ 1) $\times(n+1)$ complex matrices of determinant one. Then $U=S U(n+1)$ is a compact real form of $G$ and the Lie algebra $\mathfrak{u}=\mathfrak{s u}(n+1)$ of $U$ is equal to the subalgebra of $\mathfrak{g}$ consisting of skew-hermitian matrices of trace zero. As maximal torus $\mathfrak{t}$ in $\mathfrak{u}$ we take the $(n+1) \times(n+1)$ purely imaginary diagonal matrices of trace zero. Then $\mathfrak{h}$ consists of all diagonal matrices of trace zero and we denote by $\operatorname{diag}\left(a_{1}, a_{2}, \ldots, a_{n+1}\right) \quad\left(a_{i} \in \mathbf{C}\right)$ the diagonal matrix in $\mathfrak{h}$ with entries $a_{1}, a_{2}, \ldots, a_{n+1}$. Let $\varepsilon_{j} \in \mathfrak{h}^{*}$ be defined by $\varepsilon_{j}\left(\operatorname{diag}\left(a_{1}, \ldots, a_{n+1}\right)\right)=$ $a_{j}(j=1,2, \ldots, n+1)$. Then $\varepsilon_{1}+\cdots+\varepsilon_{n+1}=0$ as elements of $\mathfrak{h}^{*}$. Note that $\varepsilon_{j}$ is purely imaginary on $\mathrm{t}$. The set $\Delta=\left\{\varepsilon_{i}-\varepsilon_{j} \mid 1 \leq i, j \leq n+1, i \neq j\right\}$ is the collection of roots of $\mathfrak{g}$. As simple roots we take $\alpha_{i}=\varepsilon_{i}-\varepsilon_{i+1} \quad(i=$ $1,2, \ldots, n)$. The fundamental weights $\omega_{1}, \ldots, \omega_{n}$ are given by

$$
\begin{aligned}
\omega_{j} & =(n+1)^{-1}\left((n-j+1)\left(\varepsilon_{1}+\cdots+\varepsilon_{j}\right)-j\left(\varepsilon_{j+1}+\cdots+\varepsilon_{n+1}\right)\right) \\
& =\varepsilon_{1}+\cdots+\varepsilon_{j},
\end{aligned}
$$

where for the last equality we used the fact that $\varepsilon_{1}+\cdots+\varepsilon_{n+1}=0$. The Killing form on $\mathfrak{h}$ is given by $\left\langle H, H^{\prime}\right\rangle=2(n+1) \operatorname{Trace}\left(H H^{\prime}\right)$, so that $\left\langle\alpha_{i}, \alpha_{i}\right\rangle=$ $(n+1)^{-1}$. The Weyl group is isomorphic to the symmetric group $S_{n+1}$ and $\varepsilon(w)$ equals the sign of the permutation $w \in S_{n+1}$. For $P_{0}$ we can choose the set

$$
\begin{aligned}
P_{0} & =\left\{H \in \mathfrak{t} \mid \alpha_{i}(H)>0(i=1, \ldots, n), \varepsilon_{1}-\varepsilon_{n+1}(H)<2 \pi i\right\} \\
& =\left\{\operatorname{diag}\left(i a_{1}, \ldots, i a_{n+1}\right) \in \mathfrak{t} \mid a_{n+1}+2 \pi>a_{1}>a_{2}>\cdots>a_{n+1}\right\} .
\end{aligned}
$$

From the well-known realization of the finite dimensional representations $\pi_{k}$ of $S U(n+1)$ corresponding to the fundamental weights $\omega_{k}(k=1, \ldots, n)$ on the exterior powers of $\mathbf{C}^{n+1}$ (see e.g. [26, §4.7], [17, Chapter V, Example A9]) one obtains that the characters $\chi_{k}$ of $\pi_{k}$ are given by

$$
\chi_{k}(\exp H)=\sum_{1 \leq i_{1}<i_{2}<\cdots<i_{k} \leq n+1} x_{i_{1}} x_{i_{2}} \cdots x_{i_{k}} \quad(k=1, \ldots, n),
$$


where $x_{j}=e^{\varepsilon_{j}(H)}=e^{i a_{j}}$ if $H=\operatorname{diag}\left(i a_{1}, \ldots, i a_{n+1}\right) \quad\left(a_{j} \in \mathbf{R}\right)$ is in $\mathfrak{t}$. In other words, $\chi_{k}$ is the $k$ th elementary symmetric polynomial in the variables $x_{1}, \ldots, x_{n+1} \quad(k=1, \ldots, n)$. Since $a_{1}+\cdots+a_{n+1}=0(\bmod 2 \pi)$ we have $x_{1} x_{2} \cdots x_{n+1} \equiv 1$. If $\lambda$ is a dominant weight then we can write

$$
\lambda=\mu_{1} \omega_{1}+\mu_{2} \omega_{2}+\cdots+\mu_{n} \omega_{n},
$$

where $\mu_{i} \in \mathbf{Z}^{+}$( $\mathbf{Z}^{+}$denotes the set of nonnegative integers). From (2.17) we get

$$
\lambda=\lambda_{1} \varepsilon_{1}+\lambda_{2} \varepsilon_{2}+\cdots+\lambda_{n} \varepsilon_{n},
$$

where

$$
\lambda_{i}=\mu_{i}+\mu_{i+1}+\cdots+\mu_{n} \quad(i=1, \ldots, n)
$$

and

$$
\lambda_{1} \geq \lambda_{2} \geq \cdots \geq \lambda_{n} \geq 0 .
$$

For convenience we put $\lambda_{n+1}=0$ and we let $\lambda$ correspond to the partition $\left(\lambda_{1}, \lambda_{2}, \ldots, \lambda_{n+1}\right)=\left(\lambda_{1}, \lambda_{2}, \ldots, \lambda_{n}, 0\right)$ of length $\leq n$. With this notation the partition $(n, n-1, \ldots, 1,0)$ corresponds to the weight $\rho=n \varepsilon_{1}+$ $(n-1) \varepsilon_{2}+\cdots+\varepsilon_{n}=\omega_{1}+\omega_{2}+\cdots+\omega_{n}=\frac{1}{2} \sum_{\alpha>0} \alpha$, which explains the notation in subsection a. Note that $\lambda_{i}-\lambda_{i+1}=\mu_{i}$ so that $\left(\lambda_{1}, \ldots, \lambda_{n}\right)$ is precisely the dual of the partition $\left(1^{\mu_{1}} 2^{\mu_{2}} \cdots n^{\mu_{n}}\right)$. Since $a_{1}+\cdots+a_{n+1}=0(\bmod 2 \pi)$ we have

$$
\prod_{1 \leq k<l \leq n+1}\left(e^{i\left(a_{k}-a_{l}\right) / 2}-e^{i\left(a_{k}-a_{l}\right) / 2}\right)=\prod_{1 \leq k<l \leq n+1}\left(e^{i a_{k}}-e^{i a_{l}}\right),
$$

so that by Weyl's character formula [17, Chapter V, Theorem 1.7] the character $\chi_{\lambda}$ corresponding to highest weight $\lambda=\lambda_{1} \varepsilon_{1}+\cdots+\lambda_{n} \varepsilon_{n} \quad\left(\lambda_{1} \geq \cdots \geq \lambda_{n} \geq\right.$ $\left.0=\lambda_{n+1}\right)$ is precisely the function $s_{\lambda}$ defined by (2.9) if we take $x_{i} \quad(i=$ $1, \ldots, n+1)$ as above and $\lambda$ the partition $\left(\lambda_{1}, \ldots, \lambda_{n+1}\right)$. Hence

$$
\chi_{\lambda}(\exp H)=\left[\operatorname{det}\left(x_{i}^{\lambda_{j}+n+1-j}\right)_{1 \leq i, j \leq n+1}\right] /\left[\operatorname{det}\left(x_{i}^{n+1-j}\right)_{1 \leq i, j \leq n+1}\right] .
$$

In contrast to subsection a, the $n+1$ variables $x_{1}, \ldots, x_{n+1}$ are subject to the relation $x_{1} x_{2} \cdots x_{n+1}=1$. For future reference we remark that from (2.20) follows that for the weight function $\delta(\exp H)^{1 / 2}$ as given by (2.16) we have

$$
\begin{aligned}
\delta(\exp H)^{1 / 2} & =\prod_{1 \leq k<l \leq n+1}\left|e^{i\left(a_{k}-a_{l}\right) / 2}-e^{i\left(a_{k}-a_{l}\right) / 2}\right| \\
& =\prod_{1 \leq k<l \leq n+1}\left|x_{k}-x_{l}\right|,
\end{aligned}
$$

where, as before, $x_{k}=e^{i a_{k}}(k=1,2, \ldots, n+1)$ if $H=\operatorname{diag}\left(i a_{1}, \ldots, i a_{n+1}\right)$ $\left(a_{k} \in \mathbf{R}\right)$. It is worthwhile to note some special cases of the correspondence between $s_{\lambda}$ and $\chi_{\lambda}$. If $\lambda=\omega_{k}=\varepsilon_{1}+\cdots+\varepsilon_{k}$ then by (2.11) we have

$$
\chi_{\omega_{k}}=s_{\left(1^{k}\right)}=e_{k} \quad(k=1, \ldots, n),
$$


which proves (2.19) again. And if $\lambda=k \varepsilon_{1}=k \omega_{1}$, then (2.11) implies that

$$
\chi_{k: \omega_{1}}=s_{(k)}=h_{k} \text {. }
$$

\section{The RAdial PaRT OF the LAPlace-Beltrami operator}

In this section we describe a result in [4, Appendix 2] which will be crucial for the remainder of this paper. In $\mathbf{R}^{n+1}$ we have the standard basis $\varepsilon_{1}, \varepsilon_{2}, \ldots, \varepsilon_{n+1}$ and inner product $\langle\cdot, \cdot\rangle$ for which this basis is orthonormal. Let $E$ denote the hyperplane in $\mathbf{R}^{n+1}$ orthogonal to the vector $\varepsilon_{1}+\varepsilon_{2}+\cdots+\varepsilon_{n+1}$. The inner product on $\mathbf{R}^{n+1}$ induces an inner product on $E$ which we shall also denote by $\langle\cdot, \cdot\rangle$. We identify the dual $E^{*}$ of $E$ by means of this inner product. Now consider the root system $\Delta$ of type $A_{n}$ in $\mathfrak{h}_{\mathbf{R}}^{*}$ as constructed in $\S 2 . c$. We identify the Euclidean structure on $\mathfrak{h}_{\mathbf{R}}^{*}$ induced by the Killing form with the ordinary Euclidean structure on $E$ given above. Notice that the Killing form is $(2(n+1))^{-1}$ times the ordinary inner product on $E$. With this identification we have $\Delta=\left\{\varepsilon_{i}-\varepsilon_{j} \mid 1 \leq i, j \leq n+1, i \neq j\right\}$ and $\langle\alpha, \alpha\rangle=2$ for $\alpha \in \Delta$. We take simple roots, positive roots, a positive Weyl chamber, fundamental weights, etc. as in §2.c. Now if $\omega_{k}(k=1, \ldots, n)$ are the fundamental weights of the root system $\Delta$, then $2 \omega_{k}$ are the fundamental weights of the root system $\Delta_{2}=2 \Delta=\{2 \alpha \mid \alpha \in \Delta\}$ with basis $\left\{2 \alpha_{1}, \ldots, 2 \alpha_{n}\right\}$. We denote by $\Lambda_{2}$ the weight lattice of $\Delta_{2}$. Let $Z\left[\Lambda_{2}\right]$ be the group algebra over $Z$ of $\Lambda_{2}$. If $\lambda \in \Lambda_{2}$ then we write $e^{\lambda}$ for the corresponding element of $\mathbf{Z}\left[\Lambda_{2}\right]$. This group algebra $\mathrm{Z}\left[\Lambda_{2}\right]$ can also be considered as the algebra of functions on $E$ generated over $\mathbf{Z}$ by the exponentials $e^{\lambda}\left(\lambda \in \Lambda_{2}\right)$, where $e^{\lambda}(\nu)=e^{\langle\lambda, \nu\rangle}(\nu \in E)$. The Weyl group acts on $\Lambda_{2}$ and so also on $\mathbf{Z}\left[\Lambda_{2}\right]$. An element $x \in \mathbf{Z}\left[\Lambda_{2}\right]$ is called skew-invariant if $s \cdot x=\varepsilon(s) x$ for all $s \in W$, and invariant if $s \cdot x=x$ for all $s \in W$. We now define the invariant elements $z_{k}(k=1,2, \ldots, n)$ by

$$
z_{k}=\sum_{\mu \in W \cdot \omega_{k}} e^{2 \mu}
$$

where $W \cdot \omega_{k}=\left\{s \omega_{k} \mid s \in W\right\}$. It is well known (see e.g. [5, Chapter VI, no. 3.4]) that the invariant elements in $Z\left[\Lambda_{2}\right]$ are precisely the polynomials in the $z_{k}$. In particular we have that a differential operator which leaves $\mathbf{Z}\left[\Lambda_{2}\right]$ invariant can be expressed in the $z$-coordinates as differential operator with polynomial coefficients. For $m \in \mathbf{C}$ we now define a differential operator $L(m)$ (on the positive Weyl chamber) by

$$
L(m)=L_{E}+m \sum_{\alpha>0} \operatorname{coth} \alpha \partial_{\alpha},
$$

where $L_{E}$ denotes the ordinary Laplacian on $E$ and $\partial_{\alpha}$ the derivative in the direction of $\alpha$. If $m=0$ then $L(m)=L_{E}$. For certain integer values of $m$, $L(m)$ is the radial part of the Laplace-Beltrami operator on certain symmetric spaces $X$ of the noncompact type (cf. [17, Chapter II, Proposition 3.9]). For 
example, if $m=2$ then $X=S L(n+1, \mathbf{C}) / S U(n+1), n \geq 1$. In [4, Appendix 2] we determined the operator $L(m)$ explicitly in the $z$-coordinates. For convenience we state the result:

$$
\begin{aligned}
L(m)= & 4 \sum_{j=1}^{n}\left[\left(j-j^{2} /(n+1)\right) z_{j}^{2}-\sum_{r=1}^{j} 2 r z_{j-r} z_{j+r}\right] \partial_{j}^{2} \\
& +8 \sum_{1 \leq j<k \leq n}\left[(j-j k /(n+1)) z_{j} z_{k}-\sum_{r=1}^{j}(k-j+2 r) z_{j-r} z_{k+r}\right] \partial_{j} \partial_{k} \\
& +2(2 /(n+1)+m) \sum_{j=1}^{n} j(n-j+1) z_{j} \partial_{j},
\end{aligned}
$$

where $z_{j}=0$ if $j>n+1, z_{j}=1$ if $j=0$ or $j=n+1$, and we used the notation $\partial_{j}$ for $\partial / \partial z_{j}$. Instead of using the hyperbolic cotangent in (3.1) we could have defined a differential operator $L^{\prime}(m)$ (on $P_{0}$ ) using the ordinary cotangent. Then $L^{\prime}(m)$ would correspond, again for certain integer values of $m$, to the radial part $\Delta\left(L_{X}\right)$ of the Laplace-Beltrami operator $L_{X}$ on certain symmetric spaces of the compact type. One has to be careful here with the case $m=2$ where we consider $X=S U(n+1)$ as symmetric space; as explained in section 2.b, a factor 2 enters if we use the explicit expression [17, Chapter II, Proposition 3.12]. For other values of $m$ one uses [17, Chapter II, Proposition 3.11]. If we now take $z_{k}$ to be

$$
z_{k}=\sum_{\mu \in W \cdot \omega_{k}} e^{2 i \mu} \quad(k=1, \ldots, n),
$$

then this would lead to exactly the same expression (3.2) for $L^{\prime}(m)$, up to a minus sign. Of course one has to treat the case $m=2$ separately. Here we take $z_{k}$ to be defined by

$$
z_{k}=\sum_{\mu \in W \cdot \omega_{k}} e^{i \mu} \quad(k=1, \ldots, n),
$$

and so there will be a factor -4 difference with (3.2).

In [4, Appendix 2] we showed that $z_{k}$ given by (3.3) is precisely the $k$ th elementary symmetric polynomial in the variables $x_{j}=e^{i \varepsilon_{j}}(j=1,2, \ldots, n+$ 1), where we consider $\varepsilon_{j}$ as linear functional on $E$ and use the fact that $\varepsilon_{1}+\cdots+\varepsilon_{n+1}=0$ on $E$. Recall that in $\S 2$ we had $\mathfrak{t}=i \mathfrak{h}_{\mathbf{R}}$, so that if $H \in \mathfrak{t}$ then $\varepsilon_{j}(H)=i \varepsilon_{j}(H / i)$ if $\varepsilon_{j}$ is considered as element of $\mathfrak{h}^{*}$. So with our identifications we obtain from (2.19) that the $z_{k}$ in (3.3) are the characters of the fundamental representations of $S U(n+1)$, i.e. for $k=1,2, \ldots, n$ we have

$$
z_{k}(H / i)=\chi_{k}(\exp H), \quad H \in \mathfrak{t},
$$

where $H / i$ on the left-hand side is considered as element of $E$. This also follows from general theory. In fact, let $\chi_{\lambda}$ be the character of a finite dimensional 
representation of a simple Lie algebra over $\mathbf{C}$ and with highest weight $\lambda$. Let $\Pi(\lambda)$ denote the set of all weights of the representation. Suppose that $\Pi(\lambda)$ is equal to the $W$-orbit of $\lambda$, so that $\Pi(\lambda)=\{s \cdot \lambda \mid s \in W\}$. A dominant weight $\lambda \neq 0$ for which this holds is called miniscule. Since the highest weight occurs with multiplicity one, we obtain that for a miniscule weight we have

$$
\chi_{\lambda}(\exp H)=\sum_{\mu \in W \cdot \lambda} e^{\mu(H)}, \quad H \in \mathfrak{t} .
$$

For $A_{n}$ the miniscule weights are precisely all the fundamental weights $\omega_{k}$ so that we indeed get (3.4). These results can be found in [6, Chapitre VIII, $\S \S 6-7]$ where also a list of all miniscule weights for all reduced root systems is given.

The following remark will be used later on. Let $w_{0}$ denote the longest Weyl group element, i.e. the Weyl group element that sends all positive roots to negative ones. Then $\omega_{k}=w_{0}\left(-\omega_{n+1-k}\right)$ so that we obtain (cf. [4, proof Proposition 3])

$$
\bar{z}_{k}=z_{n+1-k} \quad(k=1, \ldots, n),
$$

where the bar denotes complex conjugation. This also holds more general. In fact, since $\lambda$ is purely imaginary on $t=i h_{\mathbf{R}}$, one obtains immediately from Weyl's character formula that

$$
\overline{\chi_{\lambda}}=\chi_{-w_{0} \lambda}
$$

so that (3.5) is the special case $\lambda=\omega_{k}$. This result follows also from the fact that if $\lambda$ is the highest weight of a representation, then $-w_{0} \lambda$ is the highest weight of the contragredient representation.

\section{LAguerre's emanent and Sylvester's Bezoutian}

In [2 and 3] Bacry defined a second order partial differential operator using the so-called Laguerre emanent. We now show that this operator is exactly the operator $L(2)$ in (3.2). Consider the polynomial $E(t)$ in the indeterminate $t$ as defined by (2.3), but with $n$ replaced by $n+1$, i.e.

$$
E(t)=\prod_{i=1}^{n+1}\left(1+x_{i} t\right)=\sum_{r=0}^{n+1} e_{r} t^{r}
$$

Define the polynomial $F(t, s)$ in the independent variables $t$ and $s$ by

$$
F(t, s)=\left((n+1)\left(E(t) E^{\prime}(s)-E^{\prime}(t) E(s)\right) /(t-s)\right)-E^{\prime}(t) E^{\prime}(s) .
$$

This is the polynomial that is defined by Bacry $[2,(2)],[3,(7)]$ using the so-called Laguerre emanent. The coefficients of $F(t, s)$ are homogeneous polynomials of degree two in the elementary symmetric polynomials $e_{r}(r=0, \ldots, n+1)$. We want to obtain these coefficients explicitly. First we divide the expression

$$
G(t, s)=E(t) E^{\prime}(s)-E^{\prime}(t) E(s)=\sum_{\substack{p=0,1, \ldots, n+1 \\ q=0,1, \ldots, n}}(q+1) e_{p} e_{q+1}\left(t^{p} s^{q}-t^{q} s^{p}\right)
$$


explicitly by the factor $(t-s)$. Note that terms with $p=q$ are zero. Since we also want to factor out the highest power of $s t$, we distinguish between $q \geq p$ and $q<p$. Then

$$
\begin{aligned}
G(t, s)= & \sum_{p=1}^{n+1} \sum_{q=0}^{p-1}(q+1) e_{p} e_{q+1} t^{q} s^{q}\left(t^{p-q}-s^{p-q}\right) \\
& +\sum_{p=0}^{n-1} \sum_{q=p+1}^{n}(q+1) e_{p} e_{q+1} t^{p} s^{p}\left(s^{q-p}-t^{q-p}\right) .
\end{aligned}
$$

From

$$
\frac{t^{m}-s^{m}}{t-s}=\sum_{l=0}^{m-1} t^{l} s^{m-l-1} \quad(m \geq 1)
$$

then follows that

$$
\begin{aligned}
G(t, s)= & \sum_{p=1}^{n+1} \sum_{q=0}^{p-1}(q+1) e_{p} e_{q+1} t^{q} s^{q} \sum_{l=0}^{p-q-1} t^{l} s^{p-q-l-1} \\
& -\sum_{p=0}^{n-1} \sum_{q=p+1}^{n}(q+1) e_{p} e_{q+1} t^{p} s^{p} \sum_{l=0}^{q-p-1} t^{l} s^{q-p-l-1} .
\end{aligned}
$$

In the first term of this difference we change the order of summation. Then we put

$$
T_{1}=\sum_{q=0}^{n}(q+1) e_{q+1} t^{q} s^{q} \sum_{p=q+1}^{n+1} e_{p}\left(s^{p-q-1}+t s^{p-q-2}+\cdots+t^{p-q-1}\right)
$$

and

$$
T_{2}=\sum_{p=0}^{n-1} e_{p} t^{p} s^{p} \sum_{q=p+1}^{n}(q+1) e_{q+1}\left(s^{q-p-1}+t s^{q-p-2}+\cdots+t^{q-p-1}\right),
$$

so that

$$
\left(E(t) E^{\prime}(s)-E^{\prime}(t) E(s)\right) /(t-s)=T_{1}-T_{2} .
$$

Now determine the coefficient of $t^{k} s^{l}$ in $T_{1}$ and $T_{2}$. First of all, from the symmetry in $t$ and $s$ follows that we may assume $k \leq l$. Also $l \leq n$ so that from now on we take $n \geq l \geq k \geq 0$. Since

$$
t^{k} s^{l}=t^{k} s^{k}\left(s^{l-k}\right)=t^{k-1} s^{k-1}\left(t s^{l-k-1}\right)=\cdots,
$$

we obtain that the coefficient of $t^{k} s^{l}$ in $T_{1}$ equals

$$
(k+1) e_{k+1} e_{l+1}+k e_{k} e_{l+2}+\cdots+2 e_{2} e_{l+k}+e_{1} e_{l+k+1},
$$

and in $T_{2}$ equals

$e_{k}(l+2) e_{l+2}+e_{k-1}(l+3) e_{l+3}+\cdots+e_{1}(k+l+1) e_{k+l+1}+e_{0}(k+l+2) e_{k+l+2}$, 
where, as always, $e_{k} \equiv 0$ if $k>n+1$ and $e_{0}=1$. The coefficient of $t^{k-1} s^{l-1}$ $(n+1 \geq l \geq k \geq 1)$ in $T_{1}-T_{2}$ is thus equal to

$$
\begin{aligned}
k e_{k} e_{l} & +(k-l-2) e_{k-1} e_{l+1}+(k-l-4) e_{k-2} e_{l+2}+\cdots \\
& +(-k-l+2) e_{1} e_{k+l-1}+(-k-l) e_{0} e_{k+l}
\end{aligned}
$$

Since

$$
E^{\prime}(t) E^{\prime}(s)=\sum_{k, l=1}^{n+1} k l e_{k} e_{l} t^{k-1} s^{l-1}
$$

we obtain that

$$
\begin{aligned}
& F(t, s) /(n+1) \\
& =\sum_{1 \leq k, l \leq n+1}\left[\left(k-\frac{k l}{n+1}\right) e_{k} e_{l}+\sum_{r=1}^{k}(k-l-2 r) e_{k-r} e_{l+r}\right] t^{k-1} s^{l-1} .
\end{aligned}
$$

If we compare with (3.2) then we see that if we substitute $e_{n+1}=1$ then the coefficient of $t^{k-1} s^{l-1}$ in (4.2) is exactly the coefficient of $\partial_{k} \partial_{l}$ in (3.2). Since these coefficients are used by Bacry to define the operator $\Delta_{n}[2$, IV.b], $[3,(27)]$, it follows that

$$
\frac{1}{4}(n+1) L(2)=\Delta_{n+1}+(n+2) D_{n+1},
$$

where $\Delta_{n+1}$ and $D_{n+1}$ are as in [3, (27), (28)] (in [2, IV.b] the $D_{n}$ was incorrect). Also see [1] for the case $n=2$.

Bacry pointed out to me that there was a connection between the coefficients in (4.2) and the so-called Bezoutian as defined in Weber [28, §79]. Indeed, for small values of $n$ these coefficients coincide with the examples of the Bezoutian given in $[28, \S 79]$. To define this Bezoutian we first introduce the following notation. For an arbitrary polynomial $p$ in the variable $x$ we define

$$
S[p]=\sum_{i=1}^{n+1} p\left(x_{i}\right)
$$

where, as always, $x_{1}, \ldots, x_{n+1}$ are our $n+1$ independent variables. As an example one has $S\left[x^{m}\right]=p_{m}$, the $m$ th power sum. This notation follows [28, $\S 46]$. Weber's book is the main reference for the remainder of this section. Put

$$
f_{k}=x^{k}-e_{1} x^{k-1}+\cdots+(-1)^{k-1} e_{k-1} x+(-1)^{k} e_{k}, \quad k=0,1, \ldots, n,
$$

(cf. $[28, \S 74,(4)]$ ) and define the polynomials $b_{p, q}=b_{q, p}$ of degree two in the elementary symmetric polynomials by

$$
b_{n-k, n-l}=S\left[f_{k} f_{l}\right]-(n+1)^{-1} S\left[f_{k}\right] S\left[f_{l}\right], \quad 1 \leq k, l \leq n .
$$

The bilinear form in $t=\left(t_{0}, t_{1}, \ldots, t_{n-1}\right)$ and $\tau=\left(\tau_{0}, \tau_{1}, \ldots, \tau_{n-1}\right)$ defined by

$$
\sum_{p, q=0}^{n-1} b_{p, q} t_{q} \tau_{q}
$$


is called Bezoutian in $[28, \S 79]$. It is closely related to the Bezoutian of the polynomials $p(t)$ and its derivative $p^{\prime}(t)$ as defined (more generally for any two polynomials) by Sylvester [25, §I, Art. 5-Art. 8]. Although this Bezoutian plays an important role in the classical algebraic theory of polynomials (cf. [25, 28]), we have not found an explicit expression for the $b_{p, q}$ in the literature. For small values of $n \quad\left(n=3,4\right.$ and 5) the $b_{p, q}$ are given in [28, §79] (see examples below).

Proposition. Let $b_{n-k, n-l}(1 \leq k, l \leq n)$ be defined by (4.4) and substitute $e_{n+1}=1$. Then $(-1)^{k+l} 8 b_{n-k, n-l}$ is the coefficient of $\partial_{k} \partial_{l}(1 \leq k<l \leq n)$ in (3.2), and $4 b_{n-k, n-k}$ is the coefficient of $\partial_{k}^{2}(k=1, \ldots, n)$ in (3.2).

Proof. From the Newton identities (2.7) follows that $S\left[f_{k}\right]=p_{k}-e_{1} p_{k-1}+\cdots+(-1)^{k-1} e_{k-1} p_{1}+(-1)^{k}(n+1) e_{k}=(-1)^{k}(n+1-k) e_{k}$, so

$$
S\left[f_{k}\right] S\left[f_{l}\right]=(-1)^{k+l}(n+1-k)(n+1-l) e_{k} e_{l} .
$$

Since

$$
\begin{aligned}
S\left[f_{k} f_{l}\right]= & S\left[\left(f_{k}-(-1)^{k} e_{k}\right)\left(f_{l}-(-1)^{l} e_{l}\right)\right]+(-1)^{k} e_{k} S\left[f_{l}-(-1)^{l} e_{l}\right] \\
& +(-1)^{l} e_{l} S\left[f_{k}-(-1)^{k} e_{k}\right]+(-1)^{k+l} e_{k} e_{l} S[1],
\end{aligned}
$$

and

$$
\begin{aligned}
S\left[f_{k}-(-1)^{k} e_{k}\right] & =S\left[f_{k}\right]-(-1)^{k} e_{k} S[1]=(-1)^{k}(n+1-k) e_{k}-(-1)^{k}(n+1) e_{k} \\
& =(-1)^{k+1} k e_{k},
\end{aligned}
$$

we obtain that

$$
S\left[f_{k} f_{l}\right]=S\left[\left(f_{k}-(-1)^{k} e_{k}\right)\left(f_{l}-(-1)^{l} e_{l}\right)\right]+(-1)^{k+l}(n+1-k-l) e_{k} e_{l} .
$$

From

$$
f_{k}-(-1)^{k} e_{k}=(-1)^{k-1}\left(e_{k-1} x-e_{k-2} x^{2}+\cdots+(-1)^{k-1} x^{k}\right)
$$

then follows that

$$
\begin{aligned}
S\left[f_{k} f_{l}\right]= & (-1)^{k+l} S\left[\left(\sum_{i=1}^{k}(-1)^{i-1} e_{k-i} x^{i}\right)\left(\sum_{j=1}^{l}(-1)^{j-1} e_{l-j} x^{j}\right)\right] \\
& +(-1)^{k+l}(n+1-k-l) e_{k} e_{l} .
\end{aligned}
$$

If we now compare with [4, Appendix 2, equation after (A.10)], then we see that

$$
S\left[f_{k} f_{l}\right]=(-1)^{k+l}\left(T_{k, l}+(n+1-k-l) e_{k} e_{l}\right),
$$

where $T_{k, l}$ is defined as in [4, Appendix 2]. Combining with (4.5) we obtain that

$$
\begin{aligned}
b_{n-k, n-l}= & (-1)^{k+l}\left(T_{k, l}+(n+1-k-l) e_{k} e_{l}\right. \\
& \left.-(n+1)^{-1}(n+1-k)(n+1-l) e_{k} e_{l}\right) \\
= & (-1)^{k+l}\left(T_{k, l}-(n+1)^{-1} \text { kle }_{k} e_{l}\right) .
\end{aligned}
$$


Finally we compare with $[4,(\mathrm{~A} .13)]$ to conclude that

$$
b_{n-k, n-l}=(n+1)^{-2}(-1)^{k+l} S_{k, l}
$$

with $S_{k, l}$ as defined in [4, (A.10)]. From [4, (A.3)] and the definition of $S_{k, l}$, the proposition follows.

Remark. Although there is a great similarity in the definition of the coefficients of $F(t, s)$ in (4.1) and the $b_{p, q}$ in (4.4), we have not found a simple direct proof that they are the same (the $S\left[f_{k}\right]$ are directly related to $E^{\prime}(t)$; the problem however is the $\left.S\left[f_{k} f_{l}\right]\right)$.

Examples. In the examples below we recall that $e_{0}=1$ and we substitute $e_{n+1}=$ 1. We first give $b_{n-k, n-l}$ and then the coefficient $c_{k, l}$ of $\partial_{k} \partial_{l}$ in $L(m)$.

$$
\begin{array}{rlrl}
n=1: & b_{0,0}=2^{-1}\left(e_{1}^{2}-4 e_{0} e_{2}\right) & c_{1,1} & =2\left(z_{1}^{2}-4\right) \\
n=2: & b_{1,1}=3^{-1} 2\left(e_{1}^{2}-3 e_{0} e_{2}\right) & c_{1,1}=3^{-1} 8\left(z_{1}^{2}-3 z_{2}\right) \\
b_{0,0}=3^{-1} 2\left(e_{2}^{2}-3 e_{1} e_{3}\right) & c_{2,2}=3^{-1} 8\left(z_{2}^{2}-3 z_{1}\right) \\
b_{0,1}=b_{1,0}=3^{-1}\left(9 e_{0} e_{3}-e_{1} e_{2}\right) & c_{1,2}=3^{-1} 8\left(z_{1} z_{2}-9\right) \\
\left(b_{1,1}=-3^{-1} 2 A_{0}, b_{0,0}=-3^{-1} 2 A_{2}\right. & \text { and } b_{0,1}=-3^{-1} A_{1}, \\
\text { with } A_{0}, A_{1} & \text { and } \left.A_{2} \text { as in }[28, \S 68,(3)]\right) & \\
n=3: \quad b_{2,2} & =4^{-1}\left(3 e_{1}^{2}-8 e_{0} e_{2}\right) & c_{1,1} & =3 z_{1}^{2}-8 z_{2} \\
b_{1,1} & =e_{2}^{2}-2 e_{1} e_{3}-4 e_{0} e_{4} & c_{2,2} & =4 z_{2}^{2}-8 z_{1} z_{3}-16 \\
b_{0,0} & =4^{-1}\left(3 e_{3}^{2}-8 e_{2} e_{4}\right) & c_{3,3} & =3 z_{3}^{2}-8 z_{2} \\
b_{2,1} & =2^{-1}\left(6 e_{0} e_{3}-e_{1} e_{2}\right) & c_{1,2} & =4 z_{1} z_{2}-24 z_{3} \\
b_{2,0} & =4^{-1}\left(e_{1} e_{3}-16 e_{0} e_{4}\right) & c_{1,3} & =2 z_{1} z_{3}-32 \\
b_{1,0} & =2^{-1}\left(6 e_{1} e_{4}-e_{2} e_{3}\right) & c_{2,3} & =4 z_{2} z_{3}-24 z_{1}
\end{array}
$$

(the $b_{k, l}$ are taken from $[28, \S 79,(14)]$; note that $a_{i}$ in

Weber equals $\left.(-1)^{i} e_{i}\right)$.

From $[28, \S 79]$ we also recall some facts about the determinant of the Bezoutian matrix. Let $b$ denote the symmetric matrix $\left(b_{k, l}\right)_{0 \leq k, l \leq n-1}$. Recall the Vandermonde determinant $a_{\rho}$ in (2.8) and put

$$
d=a_{\rho}^{2}=\prod_{1 \leq i<j \leq n+1}\left(x_{i}-x_{j}\right)^{2},
$$

the so-called discriminant of a polynomial with roots $x_{1}, x_{2}, \ldots, x_{n+1}$. Since $d$ is a symmetric polynomial, it can be expressed in terms of the $e_{r}$. In fact $[28, \S 79]$

$$
d=(n+1) \operatorname{det} b \text {. }
$$

Examples.

$$
\begin{aligned}
& n=1: \quad d=2 b_{0,0}=e_{1}^{2}-4 e_{0} e_{2} \quad[28, \S 50,(8)] \text {. } \\
& n=2: \quad d=3 \operatorname{det} b=e_{1}^{2} e_{2}^{2}-4 e_{0} e_{2}^{3}-4 e_{1}^{3} e_{3} \\
& +18 e_{0} e_{1} e_{2} e_{3}-27 e_{0}^{2} e_{3}^{2} \quad[28, \S 50,(10)] \text {. }
\end{aligned}
$$




\section{Chebyshev Polynomials of THE SECOND KIND}

a. Definition We return to the characters of $S L(n+1, \mathbf{C})$. Recall the definition (3.3) of the $z_{k}(k=1, \ldots, n)$, which were the elementary symmetric polynomials in the variables $x_{j}=e^{i \varepsilon_{j}}(j=1, \ldots, n+1)$. Also, they are the characters of the fundamental representations of $S L(n+1, \mathbf{C})$. As noted before, every Weyl group invariant exponential polynomial (i.e. $W$-invariant element in the group algebra $\mathbf{Z}[\Lambda]$ over the weight lattice $\Lambda$ ) is a polynomial in the $z_{k}$. In particular, any character $\chi_{\lambda}$ of $S L(n+1, \mathbf{C})$ is a polynomial in the $z_{k}$. Let us write

$$
\chi_{\lambda}=U_{\lambda}\left(z_{1}, z_{2}, \ldots, z_{n}\right)
$$

to indicate that we express $\chi_{\lambda}$ as polynomial in $z_{1}, \ldots, z_{n}$. For obvious reasons (see example below) these polynomials are called Chebyshev polynomials of the second kind. We have $U_{\omega_{k}}=z_{k}$ (cf. (2.23)).

Example. Let $n=1$ so that $G=S L(2, \mathrm{C}), U=S U(2)$ and

$$
T=\left\{\operatorname{diag}\left(e^{i \theta}, e^{-i \theta}\right) \mid \theta \in \mathbf{R}\right\} .
$$

Then $\mathfrak{t}=\{\operatorname{diag}(i \theta,-i \theta) \mid \theta \in \mathbf{R}\}$ and $\mathfrak{h}=\{\operatorname{diag}(a,-a) \mid a \in \mathbf{C}\}$. Here $\Delta^{+}$ consists of the single root $\alpha$ given by $\alpha(\operatorname{diag}(a,-a))=2 a$ and the fundamental weight $\omega$ is $\frac{1}{2} \alpha$. So if $H=\operatorname{diag}(i \theta,-i \theta) \in \mathfrak{t}$ then $z=\chi_{\omega}(\exp H)=$ Trace $H=2 \cos \theta$ and by Weyl's character formula we have for $l \in \mathbf{Z}^{+}$

$$
\begin{aligned}
\chi_{l \omega}(\exp H) & =\left(e^{(l+1) \alpha(H) / 2}-e^{-(l+1) \alpha(H) / 2}\right) /\left(e^{\alpha(H) / 2}-e^{-\alpha(H) / 2}\right) \\
& =\sin (l+1) \theta / \sin \theta,
\end{aligned}
$$

so that the $\chi_{l \omega}$ expressed in terms of $z$ lead to the classical Chebyshev polynomials of the second kind in one variable.

The above definition of the $U_{\lambda}$ is also given by Bacry [2]. Weyl's character formula $(2.21)$ shows that the definitions in [1], [3, (21)] and [14, $\S 5]$ agree with the one given here (in [14] no results on the $U_{\lambda}$ are given). Special cases were treated before by Lidl [19], Dunn and Lidl [11, 12], Eier, Dunn and Lidl [13], Ricci [23] (also see the references given there); for $n=2$ they occur as the special case $\alpha=\frac{1}{2}$ in Koornwinder [18, III-IV] (also see [24, §2]); for arbitrary $n$ they are the special case $\gamma=1$ in Debiard [7] (but no proofs are given). The fact that the definition of the $U_{\lambda}$ in $[11,12,13]$ is indeed a special case of the definition given above, follows again from (2.21). In fact, take $\lambda$ of a very special form in (2.21), namely (see (2.17) for $\omega_{k}$ )

$$
\lambda_{k, l}=(k+l) \varepsilon_{1}+l \varepsilon_{2}+\cdots+l \varepsilon_{n}=k \omega_{1}+l \omega_{n} \quad\left(k, l \in \mathbf{Z}^{+}\right) .
$$

Since $x_{1} x_{2} \cdots x_{n+1}=1$ we obtain that

and

$$
\operatorname{det}\left(x_{i}^{\lambda_{j}+n+1-j}\right)_{1 \leq i, j \leq n+1}=\operatorname{det} U_{k, l},
$$

$$
\operatorname{det}\left(x_{i}^{n+1-j}\right)_{1 \leq i, j \leq n+1}=\operatorname{det} U_{0,0} .
$$


Here $\lambda_{1}=k+l$ and $\lambda_{j}=l$ if $j>2$ and $U_{k, l}$ is as defined in [13, p. 634], which is the same as $[11,(2.10)$ for $b=1]$; the case $n=2$ occurs in $[12, \S 4]$. Consequently, the $U_{\lambda_{k, l}}\left(z_{1}, \ldots, z_{n}\right)$ are exactly the polynomials $P_{k, l}^{1 / 2}(\underline{x} ; 1)$ in in $[11,13]$, and $P_{k, l}^{1 / 2}(x, y)$ in [12, $\left.\S 4\right]$ for $n=2$. Note that only if $n=2$ the $\lambda_{k, l}\left(k, l \in \mathbf{Z}^{+}\right)$give all dominant weights. In subsection $\mathrm{d}$ we will show that for $l=0$ (so $\lambda_{k, 0}=k \omega_{1}$ ) the polynomials $U_{k \omega_{1}}$ have the same generating function as the one that is used as definition for the $f^{(k)}\left(x_{1}, \ldots, x_{n}\right)$ in [19], and that they satisfy the same recurrence relation as the one that is used as definition for the $U_{k-n+1}^{(n)}$ in [23]. Let us note here that the variables $u=$ $3 f_{0}(\theta)$ and $v=3 f_{0}(-\theta)$ in $[23,(48),(49)]$ are the first and second elementary symmetric polynomials in the $e^{\varepsilon_{j} \theta} \quad(j=1,2,3) \quad\left(\varepsilon_{j}\right.$ and $f_{0}$ as in $[23, \S \S 1-$ 2]). The polynomials $U_{\lambda}$ are also the subject of Debiard and Gaveau [10, $\S 2]$. Their coordinates $x_{1}, x_{2}, \ldots, x_{n}$ correspond to the fundamental weights $\omega_{1}, \ldots, \omega_{n}$ and again it is Weyl's character formula which shows that the $p_{\left(\mu_{1}, \ldots, \mu_{n}\right)}(X)$ as defined in $[10,(2.36)]$ corresponds to the character $\chi_{\lambda}$ with highest weight $\lambda=\mu_{1} \omega_{1}+\cdots+\mu_{n} \omega_{n}$. In [18, III-IV] Koornwinder introduced a class of orthogonal polynomials $P_{k, l}^{(\alpha)}$ in two variables and involving a parameter $\alpha>-5 / 6$. From his definition [18, III, (3.7)] it is clear that for $\alpha=\frac{1}{2}$ these polynomials coincide with the $U_{\lambda_{k, l}}\left(z_{1}, z_{2}\right)$. The interesting point in [18] is that one can generalize to arbitrary $\alpha>-5 / 6$.

We end this subsection with some simple symmetry properties of the $U_{\lambda}$. First of all, because of Weyl group invariance, we only have to describe $U_{\lambda}$ for dominant weights $\lambda$, i.e. $\lambda$ of the form $\lambda_{1} \varepsilon_{1}+\cdots+\lambda_{n} \varepsilon_{n}$ where $\lambda_{1} \geq \lambda_{2} \geq \cdots \geq$ $\lambda_{n} \geq 0$; we also write $\lambda=\left(\lambda_{1}, \ldots, \lambda_{n}, 0\right)$ or $\left(\lambda_{1}, \ldots, \lambda_{n}, \lambda_{n+1}\right)$ with $\lambda_{n+1}=0$ (cf. §2.c). In the same way we use the notations

$$
U_{\lambda}=U_{\left(\lambda_{1}, \lambda_{2}, \ldots, \lambda_{n}, 0\right)}=U_{\left(\lambda_{1}, \ldots, \lambda_{n}, \lambda_{n+1}\right)} \text {. }
$$

Now note that by (3.5) and (3.6) we have

$$
U_{\lambda}\left(z_{1}, \ldots, z_{n}\right)=\overline{U_{\lambda}\left(z_{n}, \ldots, z_{1}\right)}=U_{-w_{0} \lambda}\left(z_{n}, \ldots, z_{1}\right),
$$

where $w_{0}$ is the longest Weyl group element. If $\lambda=\lambda_{1} \varepsilon_{1}+\cdots+\lambda_{n} \varepsilon_{n}$ then $-w_{0} \lambda=\lambda_{1} \varepsilon_{1}+\left(\lambda_{1}-\lambda_{n}\right) \varepsilon_{2}+\cdots+\left(\lambda_{1}-\lambda_{2}\right) \varepsilon_{n}$, and thus we obtain

$$
U_{\left(\lambda_{1}, \ldots, \lambda_{n}, 0\right)}\left(z_{1}, \ldots, z_{n}\right)=U_{\left(\lambda_{1}, \lambda_{1}-\lambda_{n}, \ldots, \lambda_{1}-\lambda_{2}, 0\right)}\left(z_{n}, \ldots, z_{1}\right) \text {. }
$$

In the special case $\lambda=\lambda_{k, l}$ as in (5.1) this gives

$$
U_{\lambda_{k, l}}\left(z_{1}, \ldots, z_{n}\right)=U_{\lambda_{l, k}}\left(z_{n}, \ldots, z_{1}\right),
$$

which is [12, Lemma 4.3(i)] if $n=2$.

b. Differential equation. Since the characters $\chi_{\lambda}$ satisfy equation $(2.14)$, the $U_{\lambda}\left(z_{1}, \ldots, z_{n}\right)$ are eigenfunctions of the operator $-4^{-1} L(2)$ given in (3.2) (recall the remark in $\S 3$ on the factor -4$)$. Consequently

$$
L(2) U_{\lambda}\left(z_{1}, \ldots, z_{n}\right)=4(\langle\lambda, \lambda\rangle+2\langle\lambda, \rho\rangle) U_{\lambda}\left(z_{1}, \ldots, z_{n}\right),
$$


where we recall from $\S 2$ that in calculating the inner product $\langle\cdot, \cdot\rangle$, one has to write $\lambda \in \mathbf{R}^{n+1}$ as $a_{1} \varepsilon_{1}+\cdots+a_{n} \varepsilon_{n}+a_{n+1} \varepsilon_{n+1}$ with $\sum_{i=1}^{n+1} a_{i}=0$.

Example. Take $\lambda=\omega_{k}$ then $U_{\lambda}=z_{k}$ and so the eigenvalue should be

$$
4\left(\left\langle\omega_{k}, \omega_{k}\right\rangle+2\left\langle\omega_{k}, \rho\right\rangle\right) \text {. }
$$

From equation (2.17) we get $\left\langle\omega_{k}, \omega_{k}\right\rangle=(n+1)^{-1} k(n+1-k)$. Also $2 \rho=$ $n \alpha_{1}+2(n-1) \alpha_{2}+\cdots+j(n-j+1) \alpha_{j}+\cdots+n \alpha_{n}$ so that $2\left\langle\omega_{k}, \rho\right\rangle=k(n-k+1)$ and thus the eigenvalue of $z_{k}$ should be $4 k(n+1-k)\left(1+(n+1)^{-1}\right)$, which is indeed correct.

From (5.5) all results on differential equations satisfied by Chebyshev polynomials of the second kind in $[1-3,13,19]$ follow. Let us first take $\lambda_{k, l}$ as in (5.1). Then

$$
\begin{aligned}
\left\langle\lambda_{k, l}, \lambda_{k, l}\right\rangle & =k^{2}\left\langle\omega_{1}, \omega_{1}\right\rangle+2 k l\left\langle\omega_{1}, \omega_{n}\right\rangle+l^{2}\left\langle\omega_{n}, \omega_{n}\right\rangle \\
& =(n+1)^{-1} n\left(k^{2}+l^{2}\right)+(n+1)^{-1} 2 k l \\
& =(n+1)^{-1}\left(n\left(k^{2}+l^{2}\right)+2 k l\right),
\end{aligned}
$$

and since $\left\langle\omega_{i}, \alpha_{j}\right\rangle=\delta_{i j}$ we obtain that

$$
\left\langle\lambda_{k, l}, 2 \rho\right\rangle=\left\langle k \omega_{1}+l \omega_{n}, \sum_{j=1}^{n} j(n-j+1) \alpha_{j}\right\rangle=n(k+l) .
$$

Hence

$$
\left\langle\lambda_{k, l}, \lambda_{k, l}\right\rangle+2\left\langle\lambda_{k, l}, \rho\right\rangle=(n+1)^{-1}(n k(n+k+1)+n l(n+l+1)+2 k l),
$$

which is $(n+1)^{-1} b^{(1 / 2)}$ in [13, Theorem 2]. From (3.2), (5.5), and (5.7) follows [13, Theorem 2]. The special case $l=0, n=2$ gives [19, Satz 7, (40)], which again is also the case $\alpha=\frac{1}{2}$ in [18, IV, (5.17) with $n=0$ ]. The generalization to Gegenbauer polynomials in $[19, \S 4 . c]$ is the special case $[18, \mathrm{IV},(5.17)$ with $n=0]$ (see also [24, §2]). For the case $l=0, n=2$, Ricci [23, (88)] derives a second order partial differential equation for the $U_{\lambda_{k, 0}}\left(=U_{k+1}^{(2)}\right.$ in his notation) which does not seem to follow from (5.5).

We have already shown in (4.3) that $\frac{1}{4}(n+1) L(2)$ is equal to the operator $\Delta_{n+1}+(n+2) D_{n+1}$ in $[1,2,3]$. Let us show how the eigenvalue in $[1,2,3]$ follows from (5.5). The "strange formula" of Freudenthal and de Vries (see e.g. [17, Chapter V, Exercise A.4]) applied to this case gives us (recall that the Killing form is $(2(n+1))^{-1}$ times the usual inner product)

$$
\langle\rho, \rho\rangle=(n+1)\left((n+1)^{2}-1\right) / 12 .
$$

Next we recall that $[16$, Chapter III, $\S 4,(9)]$

$$
2(n+1)\langle\lambda+\rho, \lambda+\rho\rangle=\sum_{\alpha \in \Delta}\langle\alpha, \lambda+\rho\rangle^{2},
$$


and so for $\lambda+\rho=a_{1} \omega_{1}+\cdots+a_{n} \omega_{n} \in \Lambda$ we have

$$
\begin{aligned}
2(n+1) & \langle\lambda+\rho, \lambda+\rho\rangle=\sum_{i, j=1}^{n+1}\left\langle e_{i}-e_{j}, \lambda+\rho\right\rangle^{2} \\
& =\sum_{i, j=1}^{n+1}\left\langle\alpha_{i}+\cdots+\alpha_{j-1}, a_{1} \omega_{1}+\cdots+a_{n} \omega_{n}\right\rangle^{2}=\sum_{i, j=1}^{n+1}\left(a_{i}+\cdots+a_{j}\right)^{2} .
\end{aligned}
$$

In $[1,2,3]$ Bacry uses the notation $\chi_{m_{1}, m_{2}, \ldots, m_{n}, 0}$ for the character $\chi_{\lambda}$ of $S L(n+1, \mathrm{C})$ with

$\lambda+\rho=m_{1} \varepsilon_{1}+\cdots+m_{n} \varepsilon_{n}=\left(m_{1}-m_{2}\right) \omega_{1}+\left(m_{2}-m_{3}\right) \omega_{2}+\cdots+\left(m_{n}-m_{n+1}\right) \omega_{n}$,

where $m_{n+1}=0$. Hence

$(n+1)(\langle\lambda+\rho, \lambda+\rho\rangle-\langle\rho, \rho\rangle)=\frac{1}{2} \sum_{i, j=1}^{n+1}\left(m_{i}-m_{j}\right)^{2}-(n+1)^{2}\left((n+1)^{2}-1\right) / 12$,

so that (4.3) and (5.5) give us

$$
\frac{1}{4}(n+1) L(2) U_{\lambda}=\left(\frac{1}{2} \sum_{i, j=1}^{n+1}\left(m_{i}-m_{j}\right)^{2}-(n+1)^{2}\left((n+1)^{2}-1\right) / 12\right) U_{\lambda},
$$

which is Bacry's result [2, (29)]. The result as stated in [1], [2, IV.b] was incorrect. In our opinion this solves the "open problem" [2, V.1].

Another way to calculate $\langle\lambda, \lambda\rangle+2\langle\lambda, \rho\rangle$ will give us the eigenvalue ${ }^{(\Delta)} \lambda_{(\mu)}$ in [10, p. 1299]. First note that if $\lambda=\mu_{1} \omega_{1}+\cdots+\mu_{n} \omega_{n}$ then we obtain from (2.17) that

$$
\langle\lambda, \lambda\rangle=(n+1)^{-1}\left(\sum_{r=1}^{n} r(n+1-r) \mu_{r}^{2}+2 \sum_{1 \leq r<s \leq n} r(n-s+1) \mu_{r} \mu_{s}\right),
$$

which is $[10,(2.33)]$. Also

$$
\langle\lambda, \rho\rangle=(n+1)^{-1}\left(\sum_{r=1}^{n} r(n+1-r) \mu_{r}+\sum_{1 \leq r<s \leq n} r(n-s+1)\left(\mu_{r}+\mu_{s}\right)\right),
$$

since $\rho=\omega_{1}+\cdots+\omega_{n}$. Hence $\langle\lambda, \lambda\rangle+2\langle\lambda, \rho\rangle$ follows immediately.

c. Orthogonality. The fact that the Chebyshev polynomials $U_{\lambda}$ form a complete orthogonal set follows immediately from Schur's orthogonality relations and the Peter-Weyl theorem as stated in $\S 2 . b$. To obtain the weight function for the orthogonality we have to change the variables in Weyl's integration formula to $z_{1}, z_{2}, \ldots, z_{n}$. So let $H=\operatorname{diag}\left(i a_{1}, \ldots, i a_{n+1}\right) \in \mathfrak{t}$, then we want to calculate the Jacobian

$$
\left|\frac{\partial\left(z_{1}, \ldots, z_{n}\right)}{\partial\left(a_{1}, \ldots, a_{n}\right)}\right|, \quad z_{k}=\sum_{\mu \in W \cdot \omega_{k}} e^{\mu(H)} \quad(k=1, \ldots, n) .
$$


Now it is well known (see e.g. [17, Chapter III, Lemma 3.7]) that if $e_{1}, e_{2}, \ldots$, $e_{n+1}$ are the elementary symmetric polynomials in the variables $x_{1}, x_{2}, \ldots$, $x_{n+1}$ then

$$
\frac{\partial\left(e_{1}, \ldots, e_{n+1}\right)}{\partial\left(x_{1}, \ldots, x_{n+1}\right)}=\prod_{1 \leq k<l \leq n+1}\left(x_{k}-x_{l}\right) .
$$

So if we put $x_{j}=e^{i y_{j}} \quad(j=1, \ldots, n+1)$ then it follows that

$$
\left|\frac{\partial\left(e_{1}, \ldots, e_{n+1}\right)}{\partial\left(y_{1}, \ldots, y_{n+1}\right)}\right|=\left|x_{1} x_{2} \cdots x_{n+1} \prod_{1 \leq k<l \leq n+1}\left(x_{k}-x_{l}\right)\right| \text {. }
$$

Introducing the relation $y_{1}+y_{2}+\cdots+y_{n+1}=0$ gives us

$$
\left|\frac{\partial\left(e_{1}, \ldots, e_{n}\right)}{\partial\left(y_{1}, \ldots, y_{n}\right)}\right|=\prod_{1 \leq k<l \leq n+1}\left|x_{k}-x_{l}\right|,
$$

so that by $(2.22)$ we have

$$
\left|\frac{\partial\left(z_{1}, \ldots, z_{n}\right)}{\partial\left(a_{1}, \ldots, a_{n}\right)}\right|=\prod_{1 \leq k<l \leq n+1}\left|x_{k}-x_{l}\right|=\delta(\exp H)^{1 / 2},
$$

if $H=\operatorname{diag}\left(i a_{1}, \ldots, i a_{n+1}\right) \in \mathfrak{t}$ and $x_{k}=e^{i a_{k}}(k=1, \ldots, n+1)$. Furthermore, if $H \in P_{0} \quad\left(P_{0}\right.$ as in (2.18)) then $\delta(\exp H)>0$ so that we can perform the change of variables in $(2.15)$ to obtain that

$$
\int_{R} U_{\lambda}\left(z_{1}, \ldots, z_{n}\right) \overline{U_{\nu}\left(z_{1}, \ldots, z_{n}\right)} \mu\left(z_{1}, \ldots, z_{n}\right)^{1 / 2} d z_{1} \ldots d z_{n}=c \cdot \delta_{\lambda, \nu},
$$

where $c$ is a constant, $R$ is the image of $P_{0}$ under the transformation $\left(a_{1}, \ldots, a_{n}\right)$ $\rightarrow\left(z_{1}, \ldots, z_{n}\right)$ and $\mu\left(z_{1}, \ldots, z_{n}\right)$ is given by

$$
\mu\left(z_{1}, \ldots, z_{n}\right)=\prod_{1 \leq k<l \leq n+1}\left|x_{k}-x_{l}\right|^{2} .
$$

Recalling the relation (4.6) we have

$$
\mu\left(z_{1}, \ldots, z_{n}\right)=(n+1)|\operatorname{det} b|,
$$

where $b$ is the determinant of the Bezoutian matrix with $z_{n+1}=1$. This gives us the weight function $|\operatorname{det} b|^{1 / 2}$ in Bacry [2, IV.c], [3, (25)]. For the case $n=2$ see [18, Theorem 3.5]. In the context of $(5.10)$ it is worthwhile to recall $(5.2)$, (5.3).

d. Generating function and recurrence relation. As in $\S 2$.a we let $x=\left(x_{1}, \ldots\right.$, $\left.x_{n+1}\right)$ and $y=\left(y_{1}, \ldots, y_{n+1}\right)$ be two sets of variables. The elementary symmetric polynomials in $y$ will be denoted by $z_{1}, \ldots, z_{n}, z_{n+1}$. We take $z_{n+1}=$ $y_{1} y_{2} \cdots y_{n+1}=1$. Due to the relation between the $s_{\lambda}$ and the characters, as indicated in $\S 2$.c, we obtain from (2.13) the following generating function for the 
polynomials $U_{\lambda}\left(z_{1}, \ldots, z_{n}\right)$ :

$$
\prod_{1 \leq k<l \leq n+1}\left(x_{k}-x_{l}\right) \prod_{k, l=1}^{n+1}\left(1-y_{k} x_{l}\right)^{-1}=\sum_{\alpha \in\left(\mathbf{Z}^{+}\right)^{n+1}} U_{\alpha-\rho}\left(z_{1}, \ldots, z_{n}\right) x^{\alpha},
$$

where $\rho=(n, n-1, \ldots, 1,0)$ (i.e. $\left.\rho=n \varepsilon_{1}+(n-1) \varepsilon_{2}+\cdots+\varepsilon_{n}\right)$. Note that $\prod_{i=1}^{n+1}\left(1-y_{i} s\right)=\sum_{i=0}^{n+1}(-1)^{i} z_{i} s^{i}$ so that the left-hand side is indeed a generating function for polynomials in $z_{1}, \ldots, z_{n}$. If we permute the $x_{i}$ in the left-hand side of $(5.12)$ then we see that

$$
U_{\left(\alpha_{1}, \ldots, \alpha_{n+1}\right)-\rho}=\varepsilon(s) U_{\left(\alpha_{s(1)}, \ldots, \alpha_{s(n+1)}\right)-\rho}, \quad s \in S_{n+1} .
$$

And indeed, in (5.12) we are only interested in those $U_{\lambda}$ with $\lambda_{1} \geq \lambda_{2} \geq \cdots \geq$ $\lambda_{n} \geq \lambda_{n+1} \geq 0$ ( $\lambda_{i}$ integer $)$, i.e. corresponding to a partition $\left(\lambda_{1}, \lambda_{2}, \ldots, \lambda_{n+1}\right)$. Moreover, we have the symmetry (5.3). Also recall that in fact we have $\lambda_{n+1}=0$ so that we can actually take $x_{n+1}=0$ in (5.12). The generating function (5.12) is exactly the same as [2, IV.d], [3, (24)] (take $A$ in [2, IV.d] of the form $\left.\operatorname{diag}\left(y_{1}, \ldots, y_{n}\right)\right)$. We also note that (2.10) gives an expression of the $U_{\lambda}$ as determinants in the $z_{k}$. In particular we obtained that $((2.24))$

$$
\chi_{k \omega_{1}}=h_{k} .
$$

For the $h_{k}$ we had the generating function (2.4). Hence

$$
\prod_{i=1}^{n+1}\left(1-x_{i} t\right)^{-1}=\sum_{k \geq 0} U_{k \omega_{1}}\left(z_{1}, \ldots, z_{n}\right) t^{r}
$$

(which can also be obtained from $(5.12)$ by taking $x_{j}=0 \quad(j=2, \ldots, n+1)$ successively). This shows that the Chebyshev polynomials $f^{(k)}\left(z_{1}, \ldots, z_{n}\right)$ of the second kind as defined in $[19,(17)]$ coincide with the $U_{k \omega_{1}}$. The generating function (5.13) is also given in [11, Lemma 3.7] and [23, (53), (54)] and, for the case $n=2$ in [12, (4.1)] and [23, (43), (44)].

As before we put $a_{\rho}(x)=\prod\left(x_{k}-x_{l}\right)$. Then (5.12) can be written as

$$
\begin{aligned}
a_{\rho}(x) & \prod_{j=1}^{n+1}\left(1-z_{1} x_{j}+z_{2} x_{j}^{2}+\cdots+(-1)^{n+1} z_{n+1} x_{j}^{n+1}\right)^{-1} \\
& =\sum \widetilde{U}_{\left(\alpha_{1}, \ldots, \alpha_{n}, \alpha_{n+1}\right)} x_{1}^{\alpha_{1}} \cdots x_{n}^{\alpha_{n}} x_{n+1}^{\alpha_{n+1}}
\end{aligned}
$$

where the sum is over all $(n+1)$-tuples $\left(\alpha_{1}, \ldots, \alpha_{n+1}\right)$ with $\alpha_{i} \in\left(\mathbf{Z}^{+}\right)^{n+1}$ and

$$
\widetilde{U}_{\left(\alpha_{1}, \ldots, \alpha_{n}, \alpha_{n+1}\right)}=U_{\left(\alpha_{1}-n, \alpha_{2}-n+1, \ldots, \alpha_{n}-1, \alpha_{n+1}\right)} .
$$

We want to obtain recurrence relations for the $\widetilde{U}_{\alpha}$. Recall that

$$
\widetilde{U}_{\left(\alpha_{1}, \ldots, \alpha_{n+1}\right)}=\varepsilon(s) \widetilde{U}_{\left(\alpha_{s(1)}, \ldots, \alpha_{s(n+1)}\right)}, \quad s \in S_{n+1} .
$$


Since by $(2.8)$ we have

$$
a_{\rho}(x)=\sum_{s \in S_{n+1}} \varepsilon(s) \prod_{l=1}^{n+1} x_{s(l)}^{n+l-1},
$$

we obtain from $(5.12)$ that

$$
\widetilde{U}_{\left(\alpha_{1}, \ldots, \alpha_{n+1}\right)}=0 \text { if } \alpha_{1}+\cdots+\alpha_{n+1}<\frac{1}{2} n(n+1),
$$

and

$$
\begin{aligned}
\tilde{U}_{\left(\rho_{s(1)}, \ldots, \rho_{s(n+1)}\right)}=\varepsilon(s) \widetilde{U}_{\left(\rho_{1}, \ldots, \rho_{n+1}\right)} & =\varepsilon(s) \\
& \text { if } \rho_{i}=n-i+1(i=1, \ldots, n+1) .
\end{aligned}
$$

These remarks give us all $\widetilde{U}_{\left(\alpha_{1}, \ldots, \alpha_{n+1}\right)}$ with $0 \leq \alpha_{1}, \ldots, \alpha_{n+1} \leq n$. If we multiply (5.14) by $(-1)^{k} z_{k} x^{k}$ for $k=1,2, \ldots, n+1$, add these terms up and compare coefficients, then we obtain for $s=1,2, \ldots, n+1$ the recurrence relations

$$
\begin{aligned}
\sum_{r=0}^{n+1}(-1)^{r} z_{r} \widetilde{U}_{\left(\alpha_{1}, \ldots, \alpha_{s-1}, \alpha_{s}-r, \alpha_{s+1}, \ldots, \alpha_{n+1}\right)}= & \\
& \alpha_{i} \geq 0(i \neq s), \alpha_{s} \geq n+1 .
\end{aligned}
$$

The starting values are given by (5.15), (5.16) and (5.17). If we restrict to the polynomials $\widetilde{U}_{\left(\alpha_{1}, n-1, n-2, \ldots, 1,0\right)}=U_{\left(\alpha_{1}-n, 0, \ldots, 0\right)}\left(\alpha_{1} \geq 0\right)$ then it suffices to use (5.18) for $s=1$ and $\alpha_{i}=n-i+1(i \neq 1)$, together with the starting values $U_{(-p, 0, \ldots, 0)}=\delta_{p, 0}(p=0,1, \ldots, n)$. This special case gives [19, Satz 4]; it also shows that the Chebyshev polynomials of the second kind as defined by the recurrence relation [23, (3), (4)] are precisely the $U_{(k, 0, \ldots, 0)}=U_{k \omega_{1}}(k \geq 0)$ (take $r=n+1$ in $[23]$ then $\left.U_{(k, 0, \ldots, 0)}=U_{k+n-1}^{(n)}\right)$. To obtain the results in $[11,12]$ one takes the special case $\alpha_{i}=l+n-i+1$ $(i=2,3, \ldots, n)$ and $\alpha_{1}=k+l+n$ for $l \geq 0$ fixed. This leads to a recurrence relation for the polynomials $P_{k, l}^{1 / 2}=U_{(k+l, l, \ldots, l, 0)}=U_{\lambda_{k, l}}$ (cf. subsection a) which is precisely [11, Theorem 3.9] and, for the case $n=2$, [12, Theorem 4.4] (to obtain the other recurrence relation in $[11,12]$ one uses $U_{(k+l, l, \ldots, l, 0)}\left(z_{1}, \ldots, z_{n}\right)=U_{(k+l, k, k, \ldots, k, 0)}\left(z_{n}, \ldots, z_{1}\right)$, which is $\left.(5.4)\right)$. Note that in contrast to $[11,12]$ we do not need any starting values other than (5.15)-(5.17). As an example we show how the starting value $P_{1,1}^{1 / 2}$ in [11, Theorem 3.9] can be obtained. Now $P_{1,1}^{1 / 2}=U_{(2,1, \ldots, 1,0)}=\widetilde{U}_{(n+2, n, n-1, \ldots, 2,0)}$ so that we use (5.18) for $s=1, \alpha_{1}=n+2, \alpha_{i}=n-i+2(i \neq 1)$. Since by (5.15) $\widetilde{U}_{\left(\alpha_{1}, \ldots, \alpha_{n+1}\right)}=0$ if $\alpha_{i}=\alpha_{j} \quad(i \neq j)$, we obtain

$$
\widetilde{U}_{(n+2, n, n-1, \ldots, 0)}=z_{1} \widetilde{U}_{(n+1, n, n-1, \ldots, 2,0)}+(-1)^{n} z_{n+1} \widetilde{U}_{(1, n, n-1, \ldots, 2,0)} .
$$

We have

$$
\begin{aligned}
\widetilde{U}_{(n+1, n, n-1, \ldots, 2,0)}\left(z_{1}, \ldots, z_{n}\right) & =U_{(1,1, \ldots, 1,0)}\left(z_{1}, \ldots, z_{n}\right) \\
& =U_{(1,0, \ldots, 0)}\left(z_{n}, \ldots, z_{1}\right)=z_{n},
\end{aligned}
$$


where the second equality follows from (5.3) and the third one is immediate from (5.18) together with (5.16), (5.17). Furthermore, by (5.17),

$$
\widetilde{U}_{(1, n, n-1, \ldots, 2,0)}=(-1)^{n-1},
$$

so that indeed

$$
P_{1,1}^{1 / 2}=U_{(2,1, \ldots, 1,0)}=z_{1} z_{n}-z_{n+1}=z_{1} z_{n}-1 .
$$

For the case $n=2$ the polynomials $P_{i, j}^{1 / 2} \quad(0 \leq i<j \leq 2)$ in [12, Theorem 4.4] follow analogously.

\section{Chebyshev POLYNOMIALS OF THE FIRST KIND}

a. Definition. As in $\S 5$ we let $z_{k}(k=1,2, \ldots, n+1)$ be the $k$ th elementary symmetric polynomial in the variables $x_{j}=e^{i a_{j}}(j=1,2, \ldots, n+1)$ where we assume that $a_{1}+\cdots+a_{n+1}=0$ so that $z_{n+1}=x_{1} x_{2} \cdots x_{n+1}=1$. For a partition $\lambda=\left(\lambda_{1}, \lambda_{2}, \ldots, \lambda_{n}, \lambda_{n+1}\right)$ with $\lambda_{n+1}=0$ we consider the polynomials $m_{\lambda}=$ $m_{\left(\lambda_{1}, \ldots, \lambda_{n}, 0\right)}$ in $x_{1}, \ldots, x_{n}$ defined by (cf. (2.1))

$$
m_{\lambda}=m_{\lambda}\left(x_{1}, \ldots, x_{n}\right)=\sum x_{1}^{\alpha_{1}} x_{2}^{\alpha_{2}} \cdots x_{n}^{\alpha_{n}} x_{n+1}^{\alpha_{n+1}},
$$

where the sum is taken over all distinct permutations $\alpha$ of $\lambda$. We can express $m_{\lambda}$ as polynomial in the $z_{k}$ and we will write

$$
m_{\lambda}=T_{\lambda}\left(z_{1}, z_{2}, \ldots, z_{n}\right) \text {. }
$$

The $T_{\lambda}$ are called Chebyshev polynomials of the first kind. Note that (cf. (2.2))

$$
T_{(1, \ldots, 1,0, \ldots, 0)}=z_{k}(k \text { times } 1 \text { in }(1, \ldots, 1,0, \ldots, 0)) \text {. }
$$

Example. Let $n=1$ and put $\lambda_{1}=k, m_{k}=m_{(k, 0)}$ and $T_{k}=T_{(k, 0)} \quad(k>$ $0)$. Then $z_{1}=2 \cos a_{1}$, and $m_{k}\left(x_{1}\right)=x_{1}^{k}+x_{2}^{-k}=e^{i k a_{1}}+e^{-i k a_{1}}$, so that $T_{k}\left(z_{1}\right)=T_{k}\left(2 \cos a_{1}\right)=2 \cos k a_{1}$. Hence $\frac{1}{2} T_{k}\left(2 z_{1}\right)$ are the classical Chebyshev polynomials of the first kind in one variable. Note that if we do not impose the condition $x_{1} x_{2}=1$, then the $T_{k}\left(z_{1}, z_{2}\right)$ are the polynomials which express the power sums $p_{k}=x_{1}^{k}+x_{2}^{k}$ in terms of the elementary symmetric polynomials $z_{k}$.

Up to a constant the above definition is also given in Bacry [3, IV.1] and Eier and Lidl [14, §2]. Next take $\lambda$ of the special form $\lambda=\lambda_{k, l}=(k+l, l, \ldots, l, 0)$ (cf. $\S 5 . \mathrm{a})$. Since $x_{1} x_{2} \cdots x_{n+1}=1$ it then follows directly from the definition that

$$
m_{\lambda_{k, l}}=\sum_{i=1}^{n+1} \sum_{\substack{j=1 \\ j \neq i}}^{n+1} x_{i}^{k} x_{j}^{-l},
$$

which shows that the $T_{\lambda_{k, l}}(k, l \geq 0)$ correspond to $P_{k, l}^{-1 / 2}(\underline{x}, 1)$ the polynomials in [11, Definition 2.1] and, for the case $n=2, P_{k, l}^{-1 / 2}(x, y)$ in [12, 
Definition 3.1] (if $k, l>0$ then $T_{\lambda_{k, l}}=P_{k, l}^{-1 / 2}$; if $k=0$ or $l=0$ then $T_{\lambda_{k, l}}=n^{-1} P_{k, l}^{-1 / 2}$ ). If moreover $l=0$ then (cf. (2.5))

$$
m_{k}=m_{\lambda_{k, 0}}=x_{1}^{k}+\cdots+x_{n+1}^{k},
$$

so that $T_{k}=T_{\lambda_{k, 0}}$ expresses the power sums in terms of the elementary symmetric polynomials (modulo the substitution $z_{n+1}=1$ ). This also shows that the $T_{k}$ are the polynomials $g_{1}^{(k)} \quad(k \geq 0)$ as defined in [19, §2.a]. As noted before (in $\S 5 . \mathrm{a}$ ), the variables $u=3 f_{0}(\theta)$ and $v=3 f_{0}(-\theta)$ in [23, (48), (49)] are the elementary symmetric polynomials in the $x_{j}=e^{\varepsilon_{j} \theta} \quad(j=1,2,3) \quad\left(f_{0}\right.$ and $\varepsilon_{j}$ as in [23, $\left.\left.\S 1-2\right]\right)$. Furthermore, $3 f_{0}(k \theta)$ in [23, $\left.\S 2\right]$ is the $k$ th power sum, so that the $T_{k}^{(2)}$ as defined in $[23,(61)]$ are equal to the $T_{k-1}$ defined above (these remarks also imply that [23, Teorema $\mathrm{V}]$ is the statement that the power sums can be expressed as polynomials in the $z_{k}$ ). The polynomials $l_{k}$ as considered by Bacry [2, V.2], [3, IV.1] also equal $T_{k}$. For $n=2$ the $T_{\lambda}$ occur as the special case $\alpha=-\frac{1}{2}$ in Koornwinder [18, III-IV] (cf. [18, III, (3.3)]; also see the remarks in $\S 5$.a); for arbitrary $n$ they are the special case $\gamma=0$ in Debiard [7] (but no proofs are given).

We end this subsection with a simple symmetry property of the $T_{\lambda}$. Consider $\overline{m_{\lambda}}$, where the bar denotes complex conjugation. Then $\overline{m_{\lambda}}$ consists of a sum of terms of the form

$$
e^{i\left(-\lambda_{s(1)} a_{1}-\lambda_{s(2)} a_{2}-\cdots-\lambda_{s(n+1)} a_{n+1}\right)},
$$

where $s \in S_{n+1}$. Suppose $s(j)=1$, then

$$
-\lambda_{s(j)} a_{j}=-\lambda_{1} \lambda_{j}=\lambda_{1}\left(a_{1}+\cdots+a_{j-1}+a_{j+1}+\cdots+a_{n+1}\right),
$$

so that

$$
\begin{aligned}
& -\lambda_{s(1)} a_{1}-\cdots-\lambda_{s(n+1)} a_{n+1} \\
& \quad=\left(\lambda_{1}-\lambda_{s(1)}\right) a_{1}+\cdots+\left(\lambda_{1}-\lambda_{s(j)}\right) a_{j}+\cdots+\left(\lambda_{1}-\lambda_{s(n+1)}\right) a_{n+1} .
\end{aligned}
$$

Consequently,

$$
\overline{m_{\lambda}}=m_{-w_{0} \lambda},
$$

where $-w_{0} \lambda$ is the partition $\left(\lambda_{1}, \lambda_{1}-\lambda_{n}, \lambda_{1}-\lambda_{n-1}, \ldots, \lambda_{1}-\lambda_{2}, 0\right)$ (compare with §5.a). In particular we have $\frac{1}{z_{k}}=z_{n+1-k}$ (cf. (3.5)), so that we obtain

$$
\begin{aligned}
& T_{\left(\lambda_{1}, \ldots, \lambda_{n}, 0\right)}\left(z_{1}, \ldots, z_{n}\right)=\overline{T_{\left(\lambda_{1}, \ldots, \lambda_{n}, 0\right)}\left(z_{n}, \ldots, z_{1}\right)} \\
& \quad=T_{\left(\lambda_{1}, \lambda_{1}-\lambda_{n}, \ldots, \lambda_{1}-\lambda_{2}, 0\right)}\left(z_{n}, \ldots, z_{1}\right) .
\end{aligned}
$$

This result generalizes [11, Lemma 3.1] and reduces for $n=2$ to [12, Lemma 3.5]. In this context we note that the polynomials $n^{-1} P_{0, k}^{-1 / 2}=n^{-1} P_{-k, 0}^{-1 / 2} \quad(k \geq$ $0)$ in $[11,(2.8)$ for $b=1],[12, \S 3]$ are equal to $T_{(k, \ldots, k, 0)}\left(z_{1}, \ldots, z_{n}\right)=$ $T_{(k, 0, \ldots, 0)}\left(z_{n}, \ldots, z_{1}\right)$. More generally, the polynomials $P_{l, k}^{-1 / 2}$ for $k, l \leq$ 
0 correspond to $T_{(k+l, k, \ldots, k, 0)}\left(z_{1}, \ldots, z_{n}\right)=T_{(k+l, l, \ldots, l, 0)}\left(z_{n}, \ldots, z_{1}\right)$ (they are equal if $k, l<0)$.

b. Differential equation. As in the beginning of $\S 3$ we put $E=\left\{v \in \mathbf{R}^{n+1} \mid\right.$ $\left.\left\langle v, \varepsilon_{1}+\cdots+\varepsilon_{n+1}\right\rangle=0\right\}$ and we consider $\varepsilon_{j}$ as linear functional on $E$. So $\varepsilon_{j}: E \rightarrow \mathbf{R}, \varepsilon_{j}(v)=a_{j}$ if $v=\sum a_{j} \varepsilon_{j} \in E$. Also $\varepsilon_{1}+\cdots+\varepsilon_{n+1}=0$ on $E$. Since $a_{j}=-a_{1}-\cdots-a_{j-1}+n a_{j}-a_{j+1}-\cdots-a_{n+1}$, each $x_{j}$, and thus also $m_{\lambda}$, can be considered as function on $E$ by putting

$$
x_{j}(v)=e^{i\left\langle-\varepsilon_{1}-\cdots-\varepsilon_{j-1}+n \varepsilon_{j}-\varepsilon_{j+1}-\cdots-\varepsilon_{n+1}, v\right\rangle}, \quad v \in E(j=1, \ldots, n+1) .
$$

Let $\Delta_{a}$ denote the Laplacian in the coordinates $a_{1}, \ldots, a_{n+1}$, i.e.

$$
\Delta_{a}=\sum_{j=1}^{n+1} \frac{\partial^{2}}{\partial a_{j}^{2}}
$$

considered as differential operator on $E$ (cf. [4, Appendix 2]). Then it follows immediately that

$$
\Delta_{a} m_{\lambda}=-\langle\lambda, \lambda\rangle m_{\lambda}
$$

where the inner product is taken on $E$, i.e.

$$
\langle\lambda, \lambda\rangle=\sum_{j=1}^{n+1} \mu_{j}^{2} \quad \text { if } \quad \lambda=\sum_{j=1}^{n+1} \mu_{j} \varepsilon_{j} \quad \text { with } \quad \sum_{j=1}^{n+1} \mu_{j}=0 .
$$

If $\lambda=\lambda_{1} \varepsilon_{1}+\cdots+\lambda_{n} \varepsilon_{n}$ then $\mu_{j}=\left(-\lambda_{1}-\cdots-\lambda_{j-1}+n \lambda_{j}-\lambda_{j+1}-\cdots-\lambda_{n}\right) /(n+1)$ so that we obtain

$$
\langle\lambda, \lambda\rangle=\left(\lambda_{1}^{2}+\cdots+\lambda_{n}^{2}\right)-\left(\lambda_{1}+\cdots+\lambda_{n}\right)^{2} /(n+1) .
$$

For the special case $\lambda_{k, l}=(k+l) \varepsilon_{1}+l \varepsilon_{2}+\cdots+l \varepsilon_{n}$ we recover (5.6). Now the operator $\Delta_{a}$ is exactly the operator $L(0)$ as given by (3.1). In particular we get from (3.2) the expression of $\Delta_{a}=L(0)$ in the coordinates $z_{1}, \ldots, z_{n}$, where again we have to remember to put in a factor $-4^{-1}$ (cf. $\left.\S 5 . \mathrm{b}\right)$. Consequently the $T_{\lambda}$ satisfy the following differential equation.

$$
L(0) T_{\lambda}\left(z_{1}, \ldots, z_{n}\right)=4\langle\lambda, \lambda\rangle T_{\lambda}\left(z_{1}, \ldots, z_{n}\right) .
$$

Example. Take $\lambda=\omega_{k}=(1, \ldots, 1,0, \ldots, 0)(k$ times 1$)$ then

$$
T_{(1, \ldots, 1,0, \ldots, 0)}=z_{k}
$$

so the eigenvalue should be $4\left\langle\omega_{k}, \omega_{k}\right\rangle=4 k(n-k+1) /(n+1)$ (cf. $\left.\S 5 . b\right)$, which is indeed correct.

From (6.3), and the expression for $\langle\lambda, \lambda\rangle$ above, all results on differential equations satisfied by Chebyshev polynomials of the first kind in $[1,2,3,13$, 19] follow. If we take $\lambda=\lambda_{k, l}$ as above then [13, Theorem 1] follows from (3.2), (5.6) and (6.3). The special case $l=0$ and $n=2$ or 3 gives [19, (31), (32) and Satz 7, (39)]; for $n=2$ this is also the case $\alpha=-\frac{1}{2}$ in [18, IV, (5.17)]. For the case $l=0, n=2$, Ricci [23, (86)] only derives a third order 
partial differential equation for the $T_{k}=T_{\lambda_{k, 0}}\left(=T_{(k+1)}^{(2)}\right.$ in his notation), which is [18, IV, (6.6) and (6.4)] (replace $(m, n)$ in [18] by $(n-1,0))$; also see [24, $\S 2]$. In $\S 5$.b we noted that $\frac{1}{4}(n+1) L(2)$ is equal to $\Delta_{n+1}+(n+2) D_{n+1}$ in [3, III.4]. Since

$$
L(2)-L(0)=4 \sum_{j=1}^{n} j(n-j+1) z_{j} \partial_{j}=4 D_{n+1},
$$

it follows immediately that

$$
\frac{1}{4}(n+1) L(0)=\Delta_{n+1}+D_{n+1} .
$$

This gives the differential equation for the $T_{k}=T_{(k, 0, \ldots, 0)}$ in [2, V.2.b], [3, IV.1] and, for the case $n=2$, [1].

c. Orthogonality. In $\S 5 . c$ we have already shown how one can obtain the Jacobian (5.9) for the transformation $\left(a_{1}, \ldots, a_{n}\right) \rightarrow\left(z_{1}, \ldots, z_{n}\right)$ from the well-known result (5.8). We also noted that this Jacobian is positive on $P_{0}=$ $\left\{\left(a_{1}, \ldots, a_{n+1}\right) \in \mathbf{R}^{n+1} \mid \sum a_{i}=0, a_{n+1}+2 \pi>a_{1}>\cdots>a_{n+1}\right\}$. Consequently, the polynomials $T_{\lambda}$ and $T_{\nu}$ ( $\lambda$ and $\nu$ partitions, $\left.\lambda \neq \nu\right)$ are orthogonal on the transformed region $R$ with respect to the weight function $\mu\left(z_{1}, \ldots, z_{n}\right)^{-1 / 2}$, where $\mu\left(z_{1}, \ldots, z_{n}\right)$ is given by (5.11). This proves all main results in [14]. It also gives [2, V.2.c] and answers the "open problem" [2, V.2] (also see [3, IV.1]). For $n=2$ these results are contained as the special case $\alpha=-\frac{1}{2}$ in [18, III-IV].

d. Generating function and recurrence relation. Notations are as in $\S 5$.d. From (2.12) one obtains a generating function for the $T_{\lambda}$ :

$$
\prod_{k=1}^{n+1}\left(1-z_{1} x_{k}+\cdots+(-1)^{n+1} z_{n+1} x_{k}^{n+1}\right)^{-1}=\sum T_{\lambda}\left(z_{1}, \ldots, z_{n}\right) h_{\lambda}(x) .
$$

Here the sum is taken over all $(n+1)$-tuples $\lambda_{1}, \ldots, \lambda_{n+1}$ with $\lambda_{1} \geq \lambda_{2} \geq \cdots \geq$ $\lambda_{n+1} \geq 0$. We have not been able to obtain a simple recurrence relation for the $T_{\lambda}$ from (6.4). The reason is that although the $h_{\lambda}$ form a basis for the symmetric polynomials, the left-hand side of (6.4) seems to have no simple expression directly in terms of the $h_{\lambda}$. For the special case $\lambda=k \varepsilon_{1}=k \omega_{1}$ one can proceed as follows. Recall from $(6.1)$ that $m_{k}(y)=m_{(k, 0, \ldots, 0)}(y)=y_{1}^{k}+\cdots+y_{n+1}^{k}$, the $k$ th power sum $p_{k}(y)$. Also, if $E(t)=\prod_{i=1}^{n+1}\left(1+y_{i} t\right)$ (cf. (2.3)), then we obtain from (2.6) that

$$
\sum_{k \geq 1} T_{k}\left(z_{1}, \ldots, z_{n}\right) t^{k-1}=E^{\prime}(-t) / E(-t) .
$$

Since

$$
t E^{\prime}(-t)+(n+1) E(-t)=(n+1)-n z_{1} t+(n-1) z_{2} t^{2}+\cdots+(-1)^{n} z_{n} t^{n},
$$

it also follows that

$$
n+1+\sum_{k \geq 1} T_{k}\left(z_{1}, \ldots, z_{n}\right) t^{k}=\left(\sum_{j=0}^{n}(-1)^{j}(n-j+1) z_{j} t^{j}\right) / E(-t) .
$$


This gives [19, Satz 1], [11, Lemma 3.4] and, for the case $n=2,[12,(3.2)$, (3.3)] and [23, (63), (64)]. From (6.5), or equivalently from (6.6), the recurrence relations and starting values for the $T_{k}$ follow immediately. In fact,

$$
\sum_{j=0}^{k-1}(-1)^{j} z_{j} T_{k-j}=(-1)^{k-1} k z_{k}, \quad k \geq 1,
$$

where $z_{k} \equiv 0$ if $k>n+1$ and $z_{0}=z_{n+1}=1$. Of course these are just the Newton identities (2.7). This result is [19, Satz 2] (also see [11, Lemma 3.4]) and, for the case $n=2,[23,(69)]$. The Newton identities (6.7) together with (6.2) also give a recurrence relation for the $T_{(k, \ldots, k, 0)}$. In fact, if we put $z_{k} \equiv 0$ for $k<n$, then

$$
\sum_{j=0}^{k-1}(-1)^{j} z_{n-j+1} T_{(k-j, k-j, \ldots, k-j, 0)}=(-1)^{k-1} k z_{n-k+1}, \quad k \geq 1,
$$

which we rewrite as

$$
\sum_{j=1}^{n-s+1}(-1)^{s+j-1} z_{s+j} T_{(j, \ldots, j, 0)}=(-1)^{s}(n-s+1) z_{s}, \quad s \leq n .
$$

From (6.5) (or (6.6)) one can obtain generating functions for products of polynomials $T_{k}=T_{(k, 0, \ldots, 0)}$; and since we have the symmetry (6.2) one can also form products involving the polynomials $T_{k}$ and $T_{(l, \ldots, l, 0)}$. This applies in particular to the $T_{\lambda_{k, l}}=T_{(k+l, l, \ldots, l, 0)}$ for which one has the obvious relations

$$
T_{(k+l, l, \ldots, l, 0)}=T_{(k, 0, \ldots, 0)} T_{(l, \ldots, l, 0)}-\widetilde{T}_{k-l}, \quad k, l>0,
$$

where

$$
\widetilde{T}_{k-l}= \begin{cases}T_{(k-l, 0, \ldots, 0)}=T_{k-l} & \text { if } k-l>0, \\ n+1 & \text { if } k=l, \\ T_{(l-k, \ldots, l-k, 0)} & \text { if } k-l<0\end{cases}
$$

(cf. [11, Lemma 3.3], [12, Lemma 3.2]). From these relations Dunn and Lidl [11, Theorem 3.5], [12, Theorem 3.3] obtain a generating function and then recurrence relations. Of course one can also obtain the recurrence relations for the $T_{\lambda_{k, l}}$ directly from (6.9). In fact, from (6.9) and (6.7) we obtain for $k \geq 1$

$$
\sum_{j=0}^{k-1}(-1)^{j} z_{j} T_{(k-j+l, l, \ldots, l, 0)}=(-1)^{k-1} k z_{k} T_{(l, \ldots, l, 0)}-\sum_{j=0}^{k-1}(-1)^{j} z_{j} \widetilde{T}_{k-j-l} .
$$

By symmetry we may assume $k>l>0$. Put $k-l=s$, then it follows from the definition of $\widetilde{T}_{k-l}$ that

$$
\begin{aligned}
\sum_{j=0}^{k-1}(-1)^{j} z_{j} \widetilde{T}_{k-j-l}= & \left(\sum_{j=0}^{s-1}(-1)^{j} z_{j} T_{k-j-l}\right)+(-1)^{s}(n+1) z_{s} \\
& +\left(\sum_{j=1}^{l-1}(-1)^{k-l+j-1} z_{k-l+j} T_{(j, \ldots, j, 0)}\right),
\end{aligned}
$$


where we use the convention that an empty sum equals zero. Now use (6.7) with $k$ replaced by $k-l=s$. Then we obtain that

$$
\begin{aligned}
& \sum_{j=0}^{k-1}(-1)^{j} z_{j} T_{(k-j+l, l, \ldots, l, 0)} \\
& =(-1)^{k-1} k z_{k} T_{(l, l, \ldots, l, 0)}+(-1)^{k-l}(k-l-n-1) z_{k-l} \\
& \quad+\sum_{j=1}^{l-1}(-1)^{k-l+j-1} z_{k-l+j} T_{(j, \ldots, j, 0)} .
\end{aligned}
$$

If $k-l \geq n+1$ then $(6.10)$ reduces to

$$
\sum_{j=0}^{n+1}(-1)^{j} z_{j} T_{(k-j+l, l, \ldots, l, 0)}=0,
$$

and if $0<k-l<n+1$ then we can use (6.8) to rewrite (6.10) as

$$
\begin{aligned}
& \sum_{j=0}^{k-1}(-1)^{j} z_{j} T_{(k-j+l, l, \ldots, l, 0)}=(-1)^{k-1}(k-1) z_{k} T_{(l, \ldots, l, 0)} \\
& \quad+\sum_{j=1}^{n-k+1}(-1)^{k+1} z_{k+j} T_{(l+j, \ldots, l+j, 0)}
\end{aligned}
$$

which shows that $(6.11)$ holds for any $k>n+1$. In particular we obtain the recurrence relations in [11, Theorem 3.6] and [12, Theorem 3.4]. Note that the initial conditions in [11, Theorem 3.6] are the Newton identities (6.7) and (6.8).

\section{ORTHOGONAL POLYNOMIALS ASSOCIATED WITH ROOT SYSTEMS}

First we remark that a good source for results on the $U_{\lambda}$ and $T_{\lambda}$ of algebraic/combinatorial nature is Macdonald's book [20]. There is of course a great amount of literature available on characters of $S L(n+1, \mathbf{C})$ (and, more generally, on characters of complex semisimple Lie groups), all of which could be of relevance if one wants to study the special class of Chebyshev polynomials. That Chebyshev polynomials are indeed a special case of a more general class of orthogonal polynomials is the subject of this last section.

As we have seen in $\S \S 5$ and 6, Chevyshev polynomials of the first and second kind are closely related to root systems of type A. If we put

$$
P_{\lambda}^{(\alpha)}\left(z_{1}, \ldots, z_{n}\right)= \begin{cases}U_{\lambda}\left(z_{1}, \ldots, z_{n}\right) & \text { if } \alpha=\frac{1}{2}, \\ T_{\lambda}\left(z_{1}, \ldots, z_{n}\right) & \text { if } \alpha=-\frac{1}{2},\end{cases}
$$

then we can combine (5.5) and (6.3) into

$$
L(2 \alpha+1) P_{\lambda}^{(\alpha)}\left(z_{1}, \ldots, z_{n}\right)=4(\langle\lambda, \lambda\rangle+\langle\lambda, \rho(2 \alpha+1)\rangle) P_{\lambda}^{(\alpha)}\left(z_{1}, \ldots, z_{n}\right)
$$

where

$$
\rho(2 \alpha+1)=(2 \alpha+1)\left(n \varepsilon_{1}+(n-1) \varepsilon_{2}+\cdots+\varepsilon_{n}\right) .
$$


The $P_{\lambda}^{(\alpha)}$ are orthogonal on the region $R$ (see $\S \S 5 . c$ and 6.c) with respect to the weight function $\mu\left(z_{1}, \ldots, z_{n}\right)^{\alpha}$, where $\mu$ is given by (5.11). Debiard [7] (no proofs are given) generalized these results to a general parameter $\alpha>$ $-(n+1)^{-1}-\frac{1}{2}$. Also, the $P_{\lambda}^{(\alpha)}$ are eigenpolynomials of a family of $n$ algebraically independent and commuting differential operators, one of which is $L(2 \alpha+1)$; this family of commuting differential operators was also introduced by Sekiguchi [24] (also see Macdonald [21] and the references given there). The first systematic study of the $P_{\lambda}^{(\alpha)}$ for general $\alpha$ was carried out by Koornwinder [18, III-IV] for the case $n=2$ (so $\alpha>-5 / 6)$. All of these results on the $P_{\lambda}^{(\alpha)}$ contain in particular the case $\alpha= \pm \frac{1}{2}$ and hence the result (7.1). To our knowledge however, the expression (3.2) of $L(2 \alpha+1)$ in the $z$-coordinates was not known (for $n=2$ see [18, IV, (5.14)], which is also in [24, §2]); it is precisely this result that enabled us to make the connection with the results on differential equations in $[1,2,3,13,19]$.

Orthogonal polynomials can also be introduced for other types of root systems. In [18, I-II] Koornwinder treated the case $B C_{2}$ where he introduced polynomials $P^{(\alpha, \beta, \gamma)}$ involving three parameters $\alpha, \beta$ and $\gamma$. Again these polynomials are eigenfunctions of two algebraically independent and commuting differential operators. Vretare [27] gave the $P^{(\alpha, \beta, \gamma)}$ for $B C_{n} \quad(n \geq 1)$ and showed [27, Corollary 4.4] that they were eigenfunctions of the generalized radial part of the Laplace-Beltrami operator $L(\alpha, \beta, \gamma)$. Then Debiard [8], [9] showed that the $P^{(\alpha, \beta, \gamma)}$ are eigenfunctions of a family of $n$ algebraically independent and commuting differential operators, one of which is $L(\alpha, \beta, \gamma)$. The most general definition of this class of orthogonal polynomials associated with arbitrary root systems is in $[15, \S 8]$.

In the family of commuting differential operators the generalized radial part of the Laplace-Beltrami operator $L(m) \quad\left(m=\left(m_{1}, \ldots, m_{r}\right)\right.$ a certain set of parameters) always plays a decisive role (cf. [15, Proposition 8.1]). Following the same method as was used in [4, Appendix 2] to obtain (3.2), it is not very difficult to calculate $L(m)$ in the $z$-coordinates for the root system $B C_{n}$ $(n \geq 1)$. Let us state the result here. In $E=\mathbf{R}^{n}$ we have the standard basis $\varepsilon_{1}, \ldots, \varepsilon_{n}$ and inner product $\langle\cdot, \cdot\rangle$ for which this basis is orthonormal. Put $\Delta=\left\{ \pm \varepsilon_{i}, \pm 2 \varepsilon_{i}(1 \leq i \leq n), \pm \varepsilon_{i} \pm \varepsilon_{j}(1 \leq i<j \leq n)\right\}$ and take $\varepsilon_{i}-\varepsilon_{i+1}$ $(i=1, \ldots, n-1), \varepsilon_{n}$ as basis for $\Delta$. The corresponding set of positive roots will be denoted by $\Delta^{+}$. Put $\Delta_{0}=\{\alpha \in \Delta \mid 2 \alpha \notin \Delta\}$ then $\Delta_{0}$ is a root system of type $C_{n}$. Let $\omega_{1}, \ldots, \omega_{n}$ be the fundamental weights of $C_{n}$ then $\omega_{j}=\varepsilon_{1}+\cdots+\varepsilon_{j}(j=1, \ldots, n)$. Define coordinates $z_{1}, \ldots, z_{n}$ by

$$
z_{k}=\sum_{\mu \in W \cdot \omega_{k}} e^{2 \mu}, \quad k=1, \ldots, n .
$$

As usual $e^{\mu}(\mu \in E)$ is the function on $E$ which sends $\nu \in E$ to $e^{\langle\mu, \nu\rangle}$. It is easy to see that $2^{-k} z_{k} \quad(k=1, \ldots, n)$ is the $k$ th elementary symmetric polynomial in the variables $x_{1}, \ldots, x_{n}$, where $x_{j}=\cosh 2 \varepsilon_{j}$. Let $L_{E}$ denote 
the ordinary Laplacian on $E$. For $m=\left(m_{1}, m_{2}, m_{3}\right) \in \mathbf{C}^{3}$ we define the operator $L(m)$ by

$$
L(m)=L_{E}+\sum_{\alpha \in \Delta^{+}} m_{\alpha} \operatorname{coth} \alpha \partial_{\alpha}
$$

where $\partial_{\alpha}$ denotes the derivative in the direction of $\alpha$ and $m_{\varepsilon_{i}}=m_{1}, m_{2 \varepsilon_{i}}=$ $m_{2}(i=1, \ldots, n)$ and $m_{\varepsilon_{i} \pm \varepsilon_{j}}=m_{3}(1 \leq i<j \leq n)$. Write $\partial_{i}=\partial / \partial z_{i}$ $(i=1, \ldots, n) . L(m)$ has the following expression in the $z$-coordinates

$$
\begin{gathered}
L(m)=4 \sum_{j=1}^{n}\left[8 \sum_{r=1}^{j-1} r z_{j-r-1} z_{j+r-1}-2 \sum_{r=1}^{j} r z_{j-r} z_{j+r}+j z_{j}^{2}+4(j-1-n) z_{j-1}^{2}\right] \partial_{j}^{2} \\
+8 \sum_{1 \leq j<k \leq n}\left[4 \sum_{r=1}^{j-1}(k-j+2 r) z_{j-r-1} z_{k+r-1}-\sum_{r=1}^{j}(k-j+2 r) z_{j-r} z_{k+r}\right. \\
\left.+j z_{j} z_{k}+4(k-1-n) z_{j-1} z_{k-1}\right] \partial_{j} \partial_{k} \\
+2 \sum_{j=1}^{n}\left[2 j z_{j}+m_{1}\left(j z_{j}+2(n-j+1) z_{j-1}\right)+2 m_{2} j z_{j}\right. \\
\left.+m_{3}\left(j(2 n-j-1) z_{j}+4(n-j+2)(n-j+1) z_{j-2}\right)\right] \partial_{j} .
\end{gathered}
$$

Here we use the convention that $z_{j} \equiv 0$ if $j<0$ or $j>n$ and $z_{0}=1$.

\section{Examples.}

$$
n=1\left(\text { so } m_{3}=0\right): \quad L(m)=4\left(z_{1}^{2}-4\right) \partial_{1}^{2}+2\left(\left(m_{1}+2 m_{2}+2\right) z_{1}+2 m_{1}\right) \partial_{1} \text {; }
$$

put $z=4^{-1}\left(2-z_{1}\right)$, then one obtains the hypergeometric differential equation (cf. [22, Chapter II, (2.15)], [8, p. 365], [9, p. 51], [10, (4.11), (7.5)]).

$$
\begin{aligned}
n=2: \quad L(m)= & 4\left(z_{1}^{2}-2 z_{2}-8\right) \partial_{1}^{2}+8\left(z_{1} z_{2}-4 z_{1}\right) \partial_{1} \partial_{2}+8\left(z_{2}^{2}-2 z_{1}^{2}+4 z_{2}\right) \partial_{2}^{2} \\
& +\left(\left(2 m_{1}+4 m_{2}+4 m_{3}+4\right) z_{1}+8 m_{1}\right) \partial_{1} \\
& +\left(4 m_{1} z_{1}+4\left(m_{1}+2 m_{2}+m_{3}+2\right) z_{2}+16 m_{3}\right) \partial_{2} ;
\end{aligned}
$$

this is [18, II, (4.1)] if we put $z_{1}=2 u, z_{2}=4 v, m_{1}=2 \alpha-2 \beta, m_{2}=2 \beta+1$, $m_{3}=2 \gamma+1$ and divide by -4 (also see [22, Chapter II, (2.23)]; take $2 k_{1}=m_{3}$, $2 k_{2}=m_{2}, 2 k_{3}=m_{1}$ and divide by 4$)$.

Remarks. 1. In [27, p. 816], [8, p. 365], [9, Chapter I, §3], [10, (4.9)], the operator $L(m)$ is given in the coordinates $x_{1}, \ldots, x_{n}$ for arbitrary $n$.

2. If $m_{1}=0$ we obtain the $C_{n}$ case. The only miniscule weight for $C_{n}$ is $\omega_{1}$ ([6, Chapter VIII, $\S 7$, no. 3] but there is a misprint: types $B$ and $C$ are interchanged). By the remarks in $\S 2$ we obtain that $z_{1}$ should be an eigenfunction of $L\left(\left(0, m_{2}, m_{3}\right)\right)$, which is indeed the case. If $m_{3} \neq 0$ then $z_{i}(i \neq 1)$ is not an eigenfunction of $L(m)$.

Concerning the orthogonality of the $P^{(\alpha, \beta, \gamma)}$ in the $B C_{n}$ case we note that there is a close connection between the weight function for $A_{n}$ and $B C_{n}$ in 
the $z$-coordinates. Define the weight function $\mu^{(\alpha, \beta, \gamma)}\left(z_{1}, \ldots, z_{n}\right)$ (for real parameters $\alpha, \beta, \gamma$ satisfying certain conditions; see [18, I, $\S 2]$ for the case $n=2$ and [27, $\S 4],[9$, Chapter I, $\S \S 3$ and 6] for the general case) by

$$
m^{(\alpha, \beta, \gamma)}\left(x_{1}, \ldots, x_{n}\right) d x_{1} \cdots d x_{n}=\mu^{(\alpha, \beta, \gamma)}\left(z_{1}, \ldots, z_{n}\right) d z_{1} \cdots d z_{n},
$$

where

$$
m^{(\alpha, \beta, \gamma)}\left(x_{1}, \ldots, x_{n}\right)=\prod_{i=1}^{n}\left(1-x_{i}\right)^{\alpha}\left(1+x_{i}\right)^{\beta} \prod_{1 \leq i<j \leq n}\left(x_{i}-x_{j}\right)^{2 \gamma+1}
$$

(if $\alpha=\frac{1}{2}, \beta=0, \gamma=0$ then $m^{(\alpha, \beta, \gamma)}$ corresponds to the function $\delta^{1 / 2}$ given by $(2.16)$ ). As in $\S 5$.c we now use (4.6) (and in this case also (2.3)) to rewrite $\mu^{(\alpha, \beta, \gamma)}$ as

$$
\mu^{(\alpha, \beta, \gamma)}\left(z_{1}, \ldots, z_{n}\right)=c\left|\sum_{r=0}^{n}(-1)^{r} z_{r}\right|^{\alpha}\left|\sum_{r=0}^{n} z_{r}\right|^{\beta}|\operatorname{det} b|^{\gamma},
$$

where $c$ is a constant and $b$ is the Bezoutian matrix. In contrast to $\S 5 . c$ however, we do not substitute $z_{n}=1$ in $b$ (note that $n$ is shifted by one in $\S 4)$. For $n=2$ we recover [18, I, (3.12)] (also see [22, Chapter II, (2.25)]). For arbitrary $n$ we get an expression for the function $\tilde{\varphi}$ in $[9, \S 6 . \mathrm{a}]$.

Another important concept related to orthogonal polynomials associated with root systems is that of a shift operator. These are differential operators which link polynomials corresponding to different values of the parameters. They were first introduced for $B C_{2}$ by Koornwinder [18, II, §5]. For $A_{2}$ the shift operator was found by Vretare [27, $\S$ ] (see [4, $\S 4$ and 5] for some more details and references and also the $A_{3}$ case). As an example we will describe the $A_{2}$ case and apply the results to the Chebyshev polynomials $U_{\lambda}$ and $T_{\lambda}$. So we let $n=2$ in $\S \S 4$ and 5 and we put $P_{\lambda}^{(\alpha)}=P_{k, l}^{(\alpha)}$ if $\lambda=\lambda_{k, l}=(k+l) \varepsilon_{1}+l \varepsilon_{2}$. The following results can be found in $[27, \S 8]$. Define the differential operator $D_{-}^{(\alpha)}$ in $z_{1}, z_{2}$ by

$$
D_{-}^{(\alpha)}=\partial_{1}^{3}+\partial_{2}^{3}+z_{1} \partial_{1}^{2} \partial_{2}+z_{2} \partial_{1} \partial_{2}^{2}+(\alpha+5 / 2) \partial_{1} \partial_{2}
$$

Then

$$
D_{-}^{(\alpha)} P_{k, l}^{(\alpha)}=c(k, l ; \alpha) P_{k-1, l-1}^{(\alpha+1)},
$$

where $c(k, l ; \alpha)$ is a constant depending on $k, l$ and $\alpha$ (note that $\lambda_{k-1, l-1}=$ $\left.\lambda_{k, l}-\left(2 \varepsilon_{1}+\varepsilon_{2}\right)=\lambda_{k, l}-\rho\right)$. In particular

$$
D_{-}^{(-1 / 2)} T_{(k+l, l, 0)}\left(z_{1}, z_{2}\right)=c\left(k, l ;-\frac{1}{2}\right) U_{(k+l-2, l-1,0)}\left(z_{1}, z_{2}\right) .
$$

Here we have

$$
c\left(k, l ;-\frac{1}{2}\right)=\left(U_{(k+l-2, l-1,0)}(3,3) / T_{(k+l-2, l-1,0)}(3,3)\right) k^{2} l^{2}(k+l)^{2} / 12 .
$$

A systematic treatment of shift operators can be found in [22]; in particular it is shown [22, Chapter III, §3] that shift operators exist for all root systems. 
For explicit expressions for the $B C_{n}$ case we refer to [27, 9] and the references given there.

\section{ACKNOWLEDGMENT}

This paper was written during my stay from June 1987 until June 1988 at the Centre for Mathematical Analysis of the Australian National University. I would like to thank everyone at the Centre for the wonderful year they have given me.

\section{Notes added in proof.}

1. In $\S 4$ we showed that the coefficients of $t^{k-1} s^{l-1}$ in $(4.1)$, i.e. $F(t, s)$, are precisely the coefficients of $\partial_{k} \partial_{l}$ in (3.2). Our proof explicitly determined these coefficients. A direct proof is given in Proposition 2.4.2 of the following paper: K. Saito, T. Yano and J. Sekiguchi, On a certain generator system of the ring of invariants of a finite reflection group, Comm. Algebra 8 (1980), 373-408. They do not determine the coefficients explicitly. A similar result is stated for $B_{l}$.

2. In $\S 4$ we also showed indirectly (in the proposition) that the coefficients of $t^{k-1} s^{l-1}$ in $F(t, s)$ are precisely the polynomials $b_{n-k, n-l}$ in (4.4). We remarked that we could not find a simple direct proof of this result. We thank the referee for providing us with a direct proof. Let us give a brief outline. From (4.1) and

$$
E^{\prime}(s) / E(s)=\sum_{i=1}^{n+1} x_{i} /\left(1+s x_{i}\right)
$$

we have

$$
\begin{aligned}
& F(t, s) / E(t) E(s) \\
& =(n+1) \sum_{i} \frac{x_{i}^{2}}{\left(1+s x_{i}\right)\left(1+t x_{i}\right)}-\sum_{i, j} \frac{x_{i} x_{j}}{\left(1+s x_{i}\right)\left(1+t x_{j}\right)}
\end{aligned}
$$

in which the coefficient of $s^{a} t^{b}$ is

$$
g_{a b}=(-1)^{a+b}\left\{(n+1) \sum_{i} x_{i}^{a+b+2}-\sum_{i, j} x_{i}^{a+1} x_{j}^{b+1}\right\} \text {. }
$$

Hence the coefficient of $s^{p} t^{q}$ in $F(t, s)$ is

$$
\begin{aligned}
f_{p q}= & \sum_{a=0}^{p} \sum_{b=0}^{q} g_{a b} e_{p-a} e_{q-b}=(n+1) \sum_{i, a, b}(-1)^{a+b} e_{p-a} e_{q-b} x_{i}^{a+b+2} \\
& -\sum_{i, j, a, b}(-1)^{a+b} e_{p-a} e_{q-b} x_{i}^{a+1} x_{j}^{b+1}
\end{aligned}
$$

From the definition of $f_{p+1}$ one obtains

$$
\sum_{a=0}^{p}(-1)^{a} e_{p-a} x_{i}^{a+1}=(-1)^{p} f_{p+1}\left(x_{i}\right)+e_{p+1}
$$


and so

$$
\begin{aligned}
f_{p q} & =(-1)^{p+q}\left\{(n+1) \sum_{i} f_{p+1}\left(x_{i}\right) f_{q+1}\left(x_{i}\right)-\sum_{i, j} f_{p+1}\left(x_{i}\right) f_{q+1}\left(x_{j}\right)\right\} \\
& =(-1)^{p+q}\left\{(n+1) S\left[f_{p+1} f_{q+1}\right]-S\left[f_{p+1}\right] S\left[f_{q+1}\right]\right\} \\
& =(n+1)(-1)^{p+q} b_{n-p-1, n-q-1} .
\end{aligned}
$$

The last two equalities follow from the definitions of $S[\cdot]$ and the $b_{i j}$. This proof can replace the proposition in $\S 4$. Note that one does not obtain the coefficients explicitly.

3. Chebyshev polynomials of the first kind associated with root systems have also been treated recently in the paper [HW1]: M. E. Hoffman and W. D. Withers, Generalized Chebyshev polynomials associated with affine Weyl groups, Trans. Amer. Math. Soc. 308 (1988), 91-104. The "generalized cosine" in this paper coincides with the " $z$-coordinates" as used in $\S 3$ for $A_{n}$, in $\S 7$ for $B C_{n}$ and in [15 and 22] for a general root system. Let $\phi$ be the $W$-orbit of $\lambda$ ( $W$ a Weyl group) and $j_{\phi}$ as defined in [HW1, §5]. For the root system $A_{n}$ the $j_{\phi}$ are exactly the $T_{\lambda}$ from $\S 6$ a. Note that in [HW1] the term "Chebyshev polynomial" is restricted to the cases $\lambda=k \omega_{i}(i=1, \ldots, n)$ with $\omega_{i}$ the fundamental weights. For the case $A_{n}$ Theorems 5.1, 5.3 and 6.1 in [HW1] correspond to results as described in $\S 6 \mathrm{c}$. As we have indicated in $\S 7$ these results are also known for arbitrary root systems. Theorem 6.2 in [HW1] are the well-known Newton identities (2.7).

In a second paper ([HW2]: Linear characters of Weyl groups and associated multivariable orthogonal polynomials, preprint) the same authors treat Chebyshev polynomials of the second kind associated with any linear character $\chi$ of $W$. In fact these characters are only used to split the given root system $\Delta$ with Weyl group $W$ into subsets $\Delta(\chi)$ that contain one or more $W$-orbits. For the sign character e.g. One obtains all of $\Delta$. Let us indicate the relation with the material treated in our paper. For any root system $\Delta$ in a Euclidean space $E$ we define a differential operator $L(m)$ by

$$
L(m)=L_{E}+\sum_{\alpha \in \Delta^{+}} m_{\alpha} \operatorname{coth} \alpha \partial_{\alpha}
$$

(cf. (3.1) for $\Delta=A_{n}, \S 7$ for $\Delta=B C_{n}$ and [15, 22] for the general case). Here $m_{\alpha}$ are complex numbers such that $m_{\alpha}=m_{w \alpha}$ for all $w \in W$. We call $m_{\alpha}$ the multiplicity of $\alpha \in \Delta$. As in the case $A_{n}$ this operator is the radial part of the Laplace-Beltrami operator on certain symmetric spaces of the noncompact type for specific integer values of the $m_{\alpha}$. The case $m_{\alpha}=2$ for all $\alpha \in \Delta$ corresponds to the complex semisimple Lie group with root system $\Delta$. Exactly as in the case of $A_{n}$ one defines Chebyshev polynomials of the second kind as the characters of the finite dimensional irreducible representations of these complex groups. In [HW2] these are obtained by using the sign character of $W$. 
They are indexed by the dominant weights $\lambda$ and are eigenfunctions of $L(2)$ (i.e. $L(m)$ with all $m_{\alpha}=2$ ). Let us also note here that Chebyshev polynomials of the first kind are obtained by considering sums over $W$ of $e^{i w \lambda}$ (cf. $\S 6$ a where $W$ is the permutation group) and that these are obtained in [HW2] by use of the identity character of $W$. They are eigenfunctions of $L(0)$, i.e. $m_{\alpha}=0$ for all $\alpha$. Now when $\Delta$ is irreducible and has only one root length then these are the only two $W$-orbits in $\Delta$ ( $\varnothing$ is considered a $W$-orbit here). When $\Delta$ is irreducible and has two root lengths then there are two intermediate cases: $m_{\alpha}=0$ for one $W$-orbit and $m_{\alpha}=2$ for the other $W$-orbit; in [HW2] this splitting is achieved using characters of $W$. This results in two more classes of Chebyshev polynomials of the second kind. These two classes correspond to the Jacobi polynomials as defined in $[15,22]$ when the parameters are chosen appropriately (e.g. $2 k_{\alpha}$ in $[15,22]$ equals $m_{\alpha}$ ). In fact this follows immediately from $[15, \S 8]$ and the fact that these Chebyshev polynomials are orthogonal with respect to the appropriate weight function. Furthermore they are eigenfunctions of $L(m)$ with $m_{\alpha}=0$ for one $W$-orbit and $m_{\alpha}=2$ for the other $W$-orbit. The eigenvalues follow from $[15, \S 8]$. As an example one can find from [22, (2.27)] the operator $L(m)$ for $G_{2}$ which has the Chebyshev polynomials as eigenfunctions in the four cases mentioned above.

4. Chebyshev polynomials associated with $A_{n}$ are closely related to the socalled zonal polynomials that are used in multivariate statistics (cf. [21] and the references given there). More generally (cf. $\S 7$ ) the Jacobi polynomials associated with $A_{n}$ are closely related to the Jack polynomials in [21]. In fact, the relation can easily be described if one expresses both as polynomials in $z_{1}, z_{2}, \ldots, z_{n+1}\left(z_{i}\right.$ as usual). Jacobi polynomials can be obtained from Jack polynomials by the substitution $z_{n+1} \equiv 1$. When a Jacobi polynomial in the variables $z_{1}, \ldots, z_{n}$ is made into a homogeneous polynomial in $z_{1}, \ldots, z_{n}, z_{n+1}$ one obtains Jack polynomials. The normalizing constant can be obtained from results stated in [21] (we also note that $2 / \alpha$ in [21] equals $m$ ). The zonal polynomials mentioned above are special cases of Jack polynomials. They are eigenfunctions of the radial part $L_{1}(m)$ of the Laplace-Beltrami operator for $G L(n+1, \mathbf{F}) / U(n+1, \mathbf{F})$ where $\mathbf{F}=\mathbf{R}, \mathbf{C}$ or $\mathbf{H}$ and $m=1,2$ or 4 correspondingly. Jack polynomials are eigenfunctions of $L_{1}(m)$ for general $m$ (cf. $[21, \S 2])$. One can obtain $L_{1}(m)$ explicitly in the $z$-coordinates. The result is of course closely related to the case $A_{n}$. We finally mention that recently $q$-analogues of orthogonal polynomials associated with arbitrary root systems have been introduced by Macdonald (Orthogonal polynomials associated with root systems, preprint, 1988).

\section{REFERENCES}

1. H. Bacry, Generalized Chebyshev polynomials and characters of $G L(N, \mathbf{C})$ and $S L(N, \mathbf{C})$, Group Theoretical Methods in Physics, Lecture Notes in Phys., vol. 201, Springer-Verlag, Berlin, 1984. 
2. __ An application of Laguerre's emanent to generalized Chebyshev polynomials, Polynômes Orthogonaux et Applications, Lecture Notes in Math., vol. 1171, Springer-Verlag, Berlin, 1985.

3. __ Zeros of polynomials and generalized Chebyshev polynomials, Group Theoretical Methods in Physics, Nauka, Moscow, 1986.

4. R. J. Beerends, The Abel transform and shift operators, Comp. Math. 66 (1988), 145-197.

5. N. Bourbaki, Éléments de mathématique. Groupes et algèbres de Lie, Chapitres 4, 5 et 6, Hermann, Paris, 1968.

6. ___ Éléments de mathématique. Groupes et algèbres de Lie, Chapitres 7 et 8, Hermann, Paris, 1975.

7. A. Debiard, Polynômes de Tchébychev et de Jacobi dans un espace euclidien de dimension $p$, C. R. Acad. Sci. Paris 296 (1983), 529-532.

8. __ Système différentiel hypergéométrique de type $B C_{p}$, C. R. Acad. Sci. Paris 304 (1987), 363-366.

9. ___ Système différentiel hypergéométrique et parties radiales des opérateurs invariants des espaces symétriques de type $B C_{p}$, Séminaire d'Algèbre Paul Dubreil et Marie-Paule Malliavin, Lecture Notes in Math., vol. 1296, Springer-Verlag, Berlin, 1987.

10. A. Debiard and B. Gaveau, Analysis on root systems, Canad. J. Math. 39 (1987), 1281-1404.

11. K. B. Dunn and R. Lidl, Multi-dimensional generalizations of the Chebyshev polynomials. I, II, Proc. Japan Acad. 56 (1980), 154-165.

12. __ Generalizations of the classical Chebyshev polynomials to polynomials in two variables, Czechoslovak Math. J. 32 (1982), 516-528.

13. R. Eier, R. Lidl, and K. B. Dunn, Differential equations for generalized Chebyshev polynomials, Rend. Math. 14 (1981), 633-646.

14. R. Eier and R. Lidl, A class of orthogonal polynomials in $k$ variables, Math. Ann. 260 (1982), 93-99.

15. G. J. Heckman, Root systems and hypergeometric functions. II, Comp. Math. 64 (1987), 353-373.

16. S. Helgason, Differential geometry, Lie groups, and symmetric spaces, Academic Press, New York, 1978.

17. _ Groups and geometric analysis, Academic Press, Orlando, 1984.

18. T. H. Koornwinder, Orthogonal polynomials in two variables which are eigenfunctions of two algebraically independent partial differential operators, I-IV, Kon. Ned. Akad. Wet. Ser. A 77 = Indag. Math. 36 (1974), 48-66 and 357-381.

19. R. Lidl, Tschebyscheffpolynome in mehreren variabelen, J. Reine Angew. Math. 273 (1975), 178-198.

20. I. G. Macdonald, Symmetric functions and Hall polynomials, Clarendon Press, Oxford, 1979.

21. _ Commuting differential operators and zonal spherical functions, Algebraic Groups Utrecht 1986, Lecture Notes in Math., vol. 1271, Springer-Verlag, Berlin, 1987.

22. E. M. Opdam, Generalized hypergeometric functions associated with root systems, Thesis, Leiden, 1988.

23. P. E. Ricci, I polinomi di Tchebycheff in più variabili, Rend. Mat. 11 (1978), 295-327.

24. J. Sekiguchi, Zonal spherical functions on some symmetric spaces, Publ. RIMS Kyoto Univ. 12 suppl. (1977), 455-459.

25. J. J. Sylvester, On a theory of the syzygetic relations of two rational integral functions, comprising an application to the theory of Sturm's functions, and that of the greatest algebraical common measure, The Collected Mathematical Papers, vol. I, Chelsea, New York, 1973. 
26. V. S. Varadarajan, Lie groups, Lie algebras, and their representations, Prentice-Hall, Englewood Cliffs, N. J., 1974.

27. L. Vretare, Formulas for elementary spherical functions and generalized Jacobi polynomials, SIAM J. Math. Anal. 15 (1984), 805-833.

28. H. Weber, Lehrbuch der Algebra, Zweite auflage, Vieweg, Braunschweig, 1898.

Centre for Mathematical Analysis, Australian National University, GPO Box 4, Canberra ACT 2601 Australia

Current address: Department of Mathematics and Computer Science, Leiden University, P.O. Box 9512, 2300 RA Leiden, The Netherlands 